\title{
A few StePS forward in unveiling the complexity of galaxy evolution: light-weighted stellar ages of intermediate-redshift galaxies with WEAVE
}

\author{
L. Costantin ${ }^{1,2}$, A. Iovino ${ }^{1}$, S. Zibetti ${ }^{3}$, M. Longhetti ${ }^{1}$, A. Gallazzi ${ }^{3}$, A. Mercurio ${ }^{4}$, I. Lonoce ${ }^{5}$, M. Balcells ${ }^{6,7,8}$, \\ M. Bolzonella ${ }^{9}$, G. Busarello ${ }^{4}$, G. Dalton ${ }^{10,11}$, A. Ferré-Mateu ${ }^{12,13}$, R. García-Benito ${ }^{14}$, A. Gargiulo ${ }^{15}$, C. Haines ${ }^{16}$, \\ S. Jin ${ }^{10,11,17}$, F. La Barbera ${ }^{4}$, S. McGee ${ }^{18}$, P. Merluzzi ${ }^{4}$, L. Morelli ${ }^{16}$, D. N. A. Murphy ${ }^{19}$, L. Peralta de Arriba ${ }^{19}$, \\ A. Pizzella ${ }^{20,21}$, B. M. Poggianti ${ }^{21}$, L. Pozzetti ${ }^{9}$, P. Sánchez-Blázquez ${ }^{22}$, M. Talia ${ }^{9,23}$, C. Tortora ${ }^{3}$, S. C. Trager $^{17}$,
} A. Vazdekis ${ }^{7,8}$, D. Vergani ${ }^{9}$, and B. Vulcani ${ }^{21}$

(Affiliations can be found after the references)

Received 22 August 2019 / Accepted 28 September 2019

\begin{abstract}
Context. The upcoming new generation of optical spectrographs on four-meter-class telescopes, with their huge multiplexing capabilities, excellent spectral resolution, and unprecedented wavelength coverage, will provide invaluable information for reconstructing the history of star formation in individual galaxies up to redshifts of about 0.7 .

Aims. We aim at defining simple but robust and meaningful physical parameters that can be used to trace the coexistence of widely diverse stellar components: younger stellar populations superimposed on the bulk of older ones.

Methods. We produced spectra of galaxies closely mimicking data from the forthcoming Stellar Populations at intermediate redshifts Survey (StePS), a survey that uses the WEAVE spectrograph on the William Herschel Telescope. First, we assessed our ability to reliably measure both ultraviolet and optical spectral indices in galaxies of different spectral types for typically expected signal-to-noise ratios. We then analyzed such mock spectra with a Bayesian approach, deriving the probability density function of $r$-and $u$-band light-weighted ages as well as of their difference. Results. We find that the ultraviolet indices significantly narrow the uncertainties in estimating the $r$ - and $u$-band light-weighted ages and their difference in individual galaxies. These diagnostics, robustly retrievable for large galaxy samples even when observed at moderate signal-to-noise ratios, allow us to identify secondary episodes of star formation up to an age of $\sim 0.1 \mathrm{Gyr}$ for stellar populations older than $\sim 1.5 \mathrm{Gyr}$, pushing up to an age of $\sim 1$ Gyr for stellar populations older than $\sim 5 \mathrm{Gyr}$.

Conclusions. The difference between $r$-band and $u$-band light-weighted ages is shown to be a powerful diagnostic to characterize and constrain extended star-formation histories and the presence of young stellar populations on top of older ones. This parameter can be used to explore the interplay between different galaxy star-formation histories and physical parameters such as galaxy mass, size, morphology, and environment.
\end{abstract}

Key words. galaxies: evolution - galaxies: formation - galaxies: fundamental parameters - galaxies: star formation - galaxies: stellar content

\section{Introduction}

In the $\Lambda$ cold dark matter paradigm, galaxies present different physical properties as the direct consequence of the multiplicity of pathways for their formation and evolution. Photometric and spectroscopic information is widely used to trace the stellar mass content and its assembling mechanisms (Pérez-González et al. 2008; Thomas et al. 2010; Davidzon et al. 2017) and to characterize substructures in nearby galaxies (i.e., bulges, disks, and bars; Méndez-Abreu et al. 2012, 2018; de Lorenzo-Cáceres et al. 2013; Morelli et al. 2015; Costantin et al. 2017, 2018), allowing for insightful comparisons with predictions from numerical simulations in a cosmological context (Nelson et al. 2015; Schaye et al. 2015). The evolution of observed galaxy properties as a function of time contains clues on how different channels of evolution have affected the hierarchical growth of their stellar mass and which environmental effects have shaped their star-formation history (SFH; Poggianti et al. 2009; Ferré-Mateu et al. 2014; La Barbera et al. 2014; Guglielmo et al. 2019). Thus, studying the evolution of observed galaxy properties allows us to explore the processes driving the assembly history of galaxies and testing the predictions of theoretical models and numerical simulations.
In recent decades, the study of galaxies in the local Universe has greatly enriched our knowledge and understanding. Theoretical and empirical approaches are now anchored by the large, uniform, and complete spectroscopic measurements of the local Universe from the Sloan Digital Sky Survey (SDSS; York et al. 2000). Observations of colors, morphology, spectral type, and star formation of galaxies show a clear bimodal distribution, where blue star-forming late-type galaxies are separated from red quiescent early-type galaxies (Kauffmann et al. 2003; Blanton et al. 2003; Baldry et al. 2004). Many observations have shown that galaxy stellar mass is one of the most fundamental quantities that enters to predict these different galaxy properties, with a relatively minor role being played by environment (Kauffmann et al. 2004; Baldry et al. 2006; Blanton \& Moustakas 2009; Bamford et al. 2009). The bimodality is also visible in spatially resolved observations of nearby galaxies (Zibetti et al. 2017; López Fernández et al. 2018), suggesting its local and structural origin within galaxies. This marked separation of two galaxy populations persists at higher redshifts, where the relative importance of the two peaks of the bimodality changes as a consequence of the continuing decline of star formation and the ensuing galaxy migration from the blue, star-forming, to the red, passive galaxy 
sequence (Haines et al. 2017). Galaxy stellar mass is still the dominant driving factor and environment plays a secondary role (Iovino et al. 2010; Kovač et al. 2010; Hahn et al. 2015).

In this context, a key question remains pertaining to whether galaxies that quenched at early epochs remained passive since then or whether they experienced further star formation episodes (the so-called rejuvenation of galaxies). Indeed, a totally passive evolution is not entirely consistent with currently available observations, especially for massive galaxies.

A puzzling observational result keeps emerging from various data: even the most massive and apparently passive galaxies do not lead an undisturbed evolution after their star formation has stopped, but may have experienced new star formation episodes. At high redshifts $(z \sim 3)$ and towards the more recent past, semi-analytical models as well as observations from CANDELS and GAMA tell us that $31 \%$ of quiescent galaxies have experienced at least one rejuvenation event (Pandya et al. 2017). Stacked spectra of red galaxies at $z \sim 0.9$ display ages too old to be connected by simply passive evolution to local SDSS galaxies (Schiavon et al. 2006). Also, the purely passive evolution of individual massive passive galaxies at $z \sim 0.7$ would result in a tiny spread in the present-day age distribution, which is inconsistent with the observed age distribution of local massive passive galaxies (Gallazzi et al. 2014). In the redshift interval $0.6<z<1.0$, the survey LEGA-C (van der Wel et al. 2016) is as of today the best suited to trace SFH in individual galaxies, targeting approximately 3000 spectra with signal-to-noise ratios $(S / N s) \gtrsim 10 \AA^{-1}$. LEGA-C observations confirm that ages of massive galaxies $\left(M>10^{11} M_{\odot}\right)$ at $z \sim 0.8$ are inconsistent with those of their local counterparts (Wu et al. 2018; Spilker et al. 2018). In the LEGA-C sample of quiescent $z \sim 0.8$ galaxies a fraction of $\sim 16 \%$ have returned to the star-forming sequence in the interval $z \sim 0.7-1.5$, after having reached quiescence at some earlier time (Chauke et al. 2019).

As the evidence for episodes of rejuvenation within the passive galaxy sample grows, suggesting that the path to quiescence is not an undisturbed one, the possible mechanisms at work and their origin (internal, external, or a mixture of both) remain a matter of discussion. A possible solution invokes continuous residual star formation in individual quiescent galaxies, where a minority of young stars add to a base of an old stellar population (Trager et al. 2000), but external mechanisms (either HI gas accretion or mergers which bring in new gas) have also been proposed (Kaviraj et al. 2009). In this context, the intermediate redshift range $(0.3 \lesssim z \lesssim 0.7)$ offers an interesting niche of investigation: the span in cosmic time covered up to $z \sim 0.7$ is nearly half the age of the Universe, enabling the direct observation of galaxies over a significant and continuous interval of their evolutionary life. In this redshift range, a coarse estimate of the morphological type is possible with data from ground-based telescopes (Krywult et al. 2017); the fraction of galaxies located in structures like groups progressively rises as expected in a hierarchical structure formation scenario (Knobel et al. 2009), and the increase of the number of red massive passive galaxies is significant down to $z \sim 0.5$ (Gargiulo et al. 2017; Haines et al. 2017). However, the intermediate redshift regime is still largely unexplored by surveys of sufficient spectral quality, lying between the redshift ranges covered by SDSS and LEGA-C data.

The upcoming new generation of spectrographs at fourmeter-class telescopes with their extraordinarily huge and multiplexing collecting capabilities, and unprecedented wavelength coverage, offer a good opportunity to fill this observational gap and to provide spectral data comparable in quality to those obtained for the nearby Universe. A non-negligible advantage offered by the wide wavelength coverage (typically $3600 \lesssim \lambda \lesssim$ $9500 \AA$ ) is that at $z>0.3$ the near-ultraviolet region of galaxy spectra enters the observed window. This is a region where lowlevel ongoing star formation, which leaves only weak imprints in the optical galaxy spectra, may be traced unambiguously (Vazdekis et al. 2016).

In this paper, we focus on the new wide-field spectroscopic facility for the $4.2 \mathrm{~m}$ William Herschel Telescope (WHT) in the Canary Islands, WEAVE (Dalton et al. 2012; Jin et al., in prep.), and on the project StePS (Stellar Population at intermediate redshift Survey; Iovino et al., in prep.), one of the eight surveys that will be carried out during the first five years of WEAVE operations (starting in 2020). StePS aims to obtain high-resolution $(R \sim 5000)$, moderate-quality $\left(S / N \gtrsim 10 \AA^{-1}\right)$ spectra of about 25000 galaxies in the redshift range $0.3-0.7$, thus providing reliable measurements of the absorption features in the stellar continuum for a statistically large sample of galaxies (roughly ten times larger than LEGA-C). The targets are selected simply by magnitude $\left(I_{\mathrm{AB}}<20.5 \mathrm{mag}\right)$ and photometric (spectroscopic when available) redshift at $z>0.3$, thus filling the crucial range between SDSS and LEGA-C data sets. The galaxy mass targeted by StePS (computed assuming the Chabrier 2003 initial mass function) ranges from $M \sim 10^{10.2} M_{\odot}$ at $z=0.3$ to $\sim 10^{11} M_{\odot}$ at $z=0.55$ and $\sim 10^{11.3} M_{\odot}$ at $z=0.7$, the massive tail of galaxy mass distribution.

The main goal of this paper is to show how the values of light-weighted stellar ages in the two photometric SDSS $u$ and $r$ bands, and their difference, are simple but efficient tools for unveiling the presence of a younger stellar population coexisting with the bulk of an older one. To achieve this purpose, StePS expected performances are tested using realistic simulations at different redshifts and $\mathrm{S} / \mathrm{N}$. The main novelty is the use of ultraviolet indices together with more classic optical ones in the context of a full Bayesian analysis to infer simple but meaningful physical properties of the past SFH of galaxies, the so-called archaeological approach. In our simulations, we use spectral data only and focus on the use of spectral indices as opposed to the full-spectral-fitting type of analysis. Spectral indices offer the advantage that one can select highly informative features to constrain stellar population parameters, attempting to break the degeneracies among them using information defined in small portions of the galaxy spectrum. Our templates include only galaxy absorption features, assuming that the emission-line contribution has been effectively removed from our spectra. This is a sensible choice given our main science goal, that is the detection of past star-formation episodes using spectral index information for those galaxies where the emission-line contribution has already faded.

The paper is organized as follows. In Sect. 2 we describe the stellar population models and the role of light-weighted ages on different photometric bands in retrieving information on recent events of SFH in galaxies. In Sect. 3 we describe the possibility to infer differences in light-weighted ages on different photometric bands using information from pairs of individual optical and ultraviolet indices. In Sect. 4 we describe a rigorous method to build realistic observations, which closely mimic spectra that will be observed by WEAVE for StePS. In Sect. 5 we present our ability to measure spectral indices through the whole spectral range and the results of our Bayesian analysis. In Sect. 6 we summarize our results and their implications in StePS and StePS-like analyses, giving our conclusions. We adopt $H_{0}=69.6 \mathrm{~km} \mathrm{~s}^{-1} \mathrm{Mpc}^{-1}, \Omega_{\mathrm{M}}=0.286$, and $\Omega_{\Lambda}=0.714$ as cosmological parameters throughout this work (Wright 2006). 


\section{Stellar population models}

In this work, spectral models of stellar populations play a crucial, two-fold role. On the one hand, they are used as the base ingredient to generate mock StePS-like observations, with a realistic wavelength coverage and noise behavior together with a full variety of SFHs, chemical evolution histories, and dust attenuation values that well reproduce the complexity of observed galaxy spectra. On the other hand, a comprehensive library of such models is required to derive the physical parameters of interest from such mock observations via the Bayesian statistical tools described in Sect. 5.2 (see also Gallazzi et al. 2005; Zibetti et al. 2017). It is worth noting that the model libraries employed for these two purposes need not be the same. However, we opt to have them stem from the same "parent library" in order to factor out all possible systematic effects arising from the base Simple Stellar Population (SSP) model, and the mathematical prescriptions for SFHs, chemical enrichment, and dust treatment. These libraries are described in Sect. 2.1.

We focus our analysis on mean stellar ages weighted on the light in different photometric bands, in particular the SDSS $r$ and $u$ bands. We define the parameter $\Delta$ (age) as the difference between these two light-weighted ages and propose it as a simple tool to detect the presence of a younger stellar population coexisting with the bulk of an older one. We justify this choice by using basic stellar physics arguments, as well as by showing how the overall general properties of the model SFHs correlate with $\Delta$ (age), irrespective of metallicity and dust content. As we see in more detail in Sect. 5, marginalizing over metallicity and dust content broadens the probability density functions (PDFs) of the observed values of light-weighted ages and $\Delta$ (age), but without introducing major systematic offsets. In this work, the model library used does not include emission lines. We focus on the stellar content of galaxies, which can be accurately described once emission lines are properly modeled and subtracted from observed data using standard fitting algorithms, such as Gas AND Absorption Line Fitting (GANDALF; Sarzi et al. 2006).

\subsection{Modeling the complex SFH of galaxies}

Despite recent advances in our understanding of the SFHs of galaxies thanks both to improved stellar population inference techniques and to more detailed simulations of galaxy evolution, we still lack information on the detailed shape of the starformation and chemical-enrichment histories of galaxies due to the huge diversity and stochastic nature of the physical mechanisms by which they are affected. Therefore, in trying to model the SFHs of galaxies, we pursue the goal of covering the observable parameter space with as many different forms, chemicalenrichment histories, and dust attenuation properties as possible in order to take parameter degeneracy properly and fully into account. Our final aim, however, is not to extract the full complexity that enters in the building of these models from the (simulated) observations, but to focus on simple parameters and their relationship with basic, fundamental properties of galaxy SFHs. In particular, we focus on light-weighted ages in different bands and their differences, and marginalize over the "details" on which they depend. The width of their posterior PDF will give back the actual ability of constraining these parameters with a realistic, StePSlike dataset.

We base our work on the comprehensive library of 500000 models introduced by Zibetti et al. (2017), which forms our parent library. Each model is characterized by a SFH, a chemical enrichment history, and a two-component dust attenuation prescription. The base models for our libraries are the Bruzual \& Charlot (2003) SSP models in the 2016 revised version, which adopt the Chabrier (2003) initial mass function, updated evolutionary tracks (Girardi et al. 2000; Marigo et al. 2013), and the MILES stellar spectral library (Sánchez-Blázquez et al. 2006; Falcón-Barroso et al. 2011, $2.5 \AA$ FWHM resolution), extended in the ultraviolet $(911 \lesssim \lambda \lesssim 3500 \AA)$, and the theoretical highresolution ( $F W H M=1 \AA$ ) models of Martins et al. (2005).

The SFHs are modeled as a superposition of a continuous or "secular" component and stochastic bursts. The secular component is described by a Sandage (1986) law:

$\operatorname{SFR}_{\tau}(t) \propto \frac{t}{\tau} \exp \left(-\frac{t^{2}}{2 \tau^{2}}\right)$

allowing for both an increasing and decaying phase for the starformation rate (SFR; see Fig. 1), the delay time and steepness of which are regulated by $\tau$ in the time interval from $t=0$ to $t_{\mathrm{obs}}$, which is randomly generated from a uniform logarithmic distribution from $5 \times 10^{8} \mathrm{yr}$ up to $\sim 1.7 \times 10^{10} \mathrm{yr}$. The value of $\tau$ is generated as a random number multiplied by $t_{\mathrm{obs}}$, so that $\tau$ can vary between $1 / 50 \times t_{\text {obs }}$ (almost an instantaneous burst) and $2 \times t_{\text {obs }}$ (resulting in a still monotonically increasing SFR at $\left.t_{\mathrm{obs}}\right)$. Overall, the distribution of the mean age of stars integrated between 0 and $t_{\text {obs }}$ is almost uniform in logarithm between $3 \times 10^{8} \mathrm{yr}$ and $8 \times 10^{9} \mathrm{yr}$ and drops to zero at $\sim 1.5 \times 10^{8} \mathrm{yr}$ and $\sim 1.7 \times 10^{10} \mathrm{yr}$. Our choice of the classical Sandage law for describing SFHs is justified by observational evidence that the majority of galaxies display first a rising and then a declining phase in their SFH, irrespective of their specific parametrization, as suggested by the integrated view of galaxy evolution and more recent simulations (Behroozi et al. 2013; Gladders et al. 2013; Abramson et al. 2015; Pacifici et al. 2016; Diemer et al. 2017; López Fernández et al. 2018).

In two-thirds of the models, random bursts are superimposed onto the secular SFH, in order to also include short-lived features in SFHs. Up to six bursts can occur, with a total mass formed in these episodes ranging between 1/1000 and two times the total stellar mass formed in the secular component. The age of each burst (i.e., the look-back time at which they occur) is randomly generated from a log-uniform distribution in the range $10^{5} \mathrm{yr}$ to $t_{\text {obs }}$. For the youngest bursts (age $<10^{7} \mathrm{yr}$ ), we allow only a reduced mass fraction to be formed, in order to avoid models whose light is completely dominated by the burst. The presence of random bursts of star formation greatly alleviates the relative rigidity of the simple parametric form chosen to reproduce the secular component, enabling us to model the presence of sharp transitions in SFHs.

Figures 1 and 2 present two sketches of example SFHs, that is, SFR as a function of time. We use $t_{\text {secular }}^{\mathrm{LB}}$ to denote the lookback time from $t_{\mathrm{obs}}$ to the peak of the secular SFH and $t_{\text {latest }}^{\mathrm{LB}}$ the look-back time from $t_{\text {obs }}$ to the latest peak of star formation. In general $t_{\text {latest }}^{\mathrm{LB}}<t_{\text {secular }}^{\mathrm{LB}}$, but if a galaxy either experienced no secondary events of star formation or such a secondary event happened before the peak of the secular star formation, then $t_{\text {secular }}^{\mathrm{LB}}=t_{\text {latest }}^{\mathrm{LB}}$.

In our models, we allow for chemical enrichment to occur along the SFH. We randomly generate the initial and final metallicity as well as a parameter describing how quickly the enrichment occurs as a function of the fraction of formed mass over the total, as detailed in Zibetti et al. (2017). Finally, we also apply a two-component dust attenuation to the spectra, following the formalism of Charlot \& Fall (2000), with the random parameter distribution described in Zibetti et al. (2017).

In order to provide a homogeneous and fine sampling of the PDFs over the whole space of observable parameters, we 


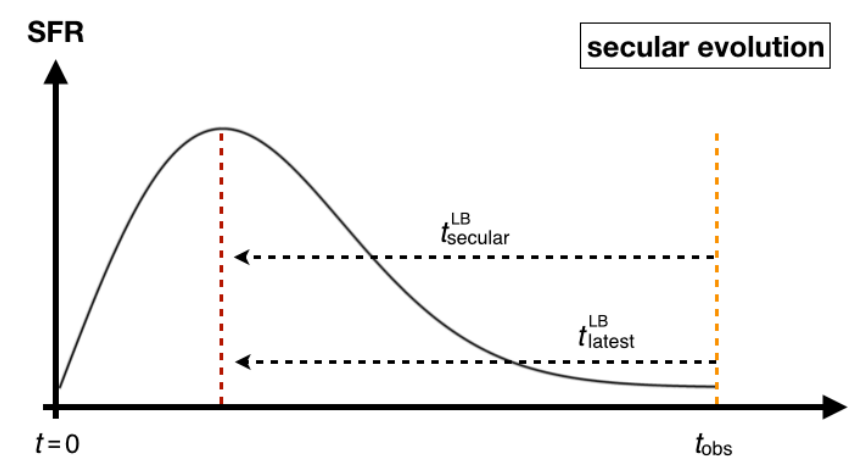

Fig. 1. Sketch of secular SFH of a galaxy that does not display a secondary burst event. The orange dashed line corresponds to the time from the beginning of the SFH $(t=0)$ to the time at which the galaxy was observed $\left(t_{\mathrm{obs}}\right)$, while the red dashed line corresponds to the peak of the secular SFH. Since the galaxy experienced no burst events, the lookback time to the peak of the secular SFH coincides with the look-back time to the latest peak of star formation $\left(t_{\text {secular }}^{\mathrm{LB}}=t_{\text {latest }}^{\mathrm{LB}}\right)$.

equalize the library to have a roughly constant density of models in the plane of $\mathrm{H} \delta+\mathrm{H} \gamma$ versus $\mathrm{D}_{\mathrm{n}} 4000$. This is done by building an initial library of 5000000 objects and selecting a subsample of 500000 so as to obtain an equalized distribution.

The various prescriptions described so far result in a roughly uniform distribution in the $r$-band light-weighted mean log age$Z$ plane, over the range 8.5-10.2 in $\log \left(\right.$ age $\left.\mathrm{yr}^{-1}\right)$ and 0.02 and 2.5 times solar in metallicity.

This combination of "secular" SFH and bursts allows us to generate a complete coverage of the observed parameter space of optical stellar absorption indices (such as $\mathrm{D}_{\mathrm{n}} 4000$, the Balmer Lick indices, and various metal-sensitive absorption indices), as we verified by comparison with the observed distribution of spatially resolved regions in CALIFA and with integrated measurements in the SDSS. Notably, with our models we can reproduce and populate the region of $\mathrm{D}_{\mathrm{n}} 4000 \lesssim 1.5$ and extremely low $\mathrm{H} \delta$, which is characterized by the "frosting" of a tiny mass fraction of a young (age $<500 \mathrm{Myr}$ ) stellar population on top of an older, mass-dominant one (see Sect. 3). This is particularly relevant in light of our attempt to identify stellar populations with a large spread in age among its components.

From this rich parent library, we randomly select a small but statistically significant chunk of 12500 models, which are used as the base to generate mock "StePS-like" observations (see Sect. 4.1). In reality, observed spectra appear broadened with different line-of-sight velocity distributions (or, simplifying, velocity dispersion). To properly retrieve physical quantities from spectral indices analysis, we convolve the originalresolution models in order to match the estimates of velocity dispersion object by object. For simplicity, we assume here the same velocity dispersion for our mock observations and we convolve both the mocks and the models with a fixed velocity dispersion of $150 \mathrm{~km} \mathrm{~s}^{-1}$. We note that the results of the analysis are not affected by the particular choice of velocity dispersion since the sensitivity of the spectral indices to the physical parameters of interest does not change greatly in the range of velocity dispersions foreseen for the galaxy masses targeted by StePS (provided that the data and the model are compared consistently).

\subsection{Light-weighted ages}

Ideally, the full reconstruction of the SFH is the goal of the stellar population analysis of a galaxy. Unfortunately, despite numer-

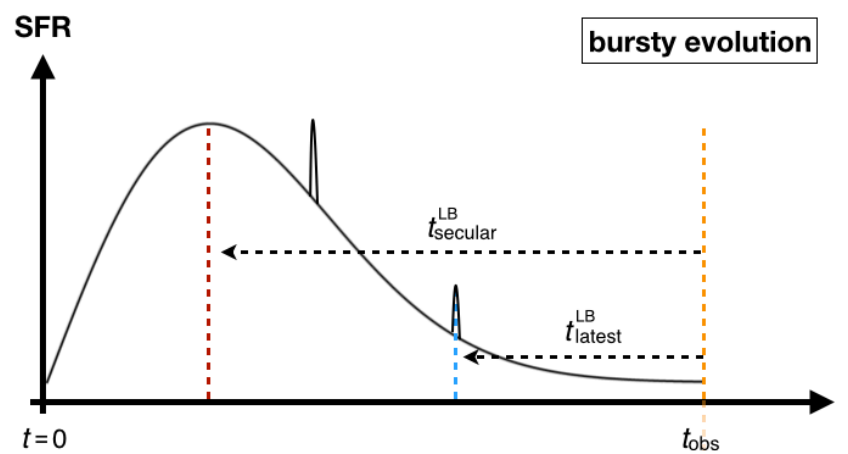

Fig. 2. Sketch of bursty SFH of a galaxy that displays additional burst events after the peak of the secular SFH. The orange dashed line corresponds to the time from the beginning of the SFH $(t=0)$ to the time at which the galaxy was observed $\left(t_{\mathrm{obs}}\right)$, the blue dashed line stands for the latest additional event of star formation superimposed to the secular SFH, and the red dashed line corresponds to the peak of the secular $\mathrm{SFH}$. The look-back time to the peak of the secular SFH ( $\left.t_{\text {secular }}^{\mathrm{LB}}\right)$ in this case differs from the look-back time to the latest peak of star formation $\left(t_{\text {latest }}^{\mathrm{LB}}\right)$.

ous attempts in the literature (e.g., ppxf: Cappellari \& Emsellem 2004, starlight: Cid Fernandes et al. 2005, steckmap: Ocvirk et al. 2006, vespa: Tojeiro et al. 2009, prospector: Leja et al. 2017, bagpipes: Carnall et al. 2018) this goal has not yet been achieved. One reason for this is the relatively low $\mathrm{S} / \mathrm{N}$ usually achievable in spectra of typical surveys at intermediate redshift, an issue typically addressed by the use of stacked spectra. Moreover, the full reconstruction of the SFH of a galaxy turns out to be an ill-posed inversion problem, hampered by a large number of degeneracies (see e.g., Ocvirk et al. 2006; Cappellari 2017). Vice versa, the lower moments of the age distribution of the stars in a galaxy, and the mean in particular, are more easily constrained by the observations and their intrinsic degeneracy-driven uncertainties can be robustly quantified by means of model libraries such as the one presented in the previous section. As demonstrated and discussed for example in Gallazzi et al. (2005) and Zibetti et al. (2017), with optical rest-frame spectra having $S / N \gtrsim 10$ one can obtain uncertainties on $r$-band light-weighted mean stellar ages of the order of $\pm 0.1-0.2$ dex based on five indices $\left(D_{n} 4000, \mathrm{H} \beta, \mathrm{H} \gamma+\right.$ $\mathrm{H} \delta,\left[\mathrm{Mg}_{2} \mathrm{Fe}\right]$, and $\left.[\mathrm{Mg} \mathrm{Fe}]^{\prime}\right)$, plus possibly broadband optical SDSS ugriz fluxes; uncertainties on age vary little with galaxy spectral type, though with a tendency of smaller relative uncertainties for older galaxies.

Here we specifically refer to light-weighted ages, defined as

age $=\frac{\int_{t=0}^{t_{\mathrm{obs}}}\left(t_{\mathrm{obs}}-t\right) \operatorname{SFR}(t) L(t) \mathrm{d} t}{\int_{t=0}^{t_{\mathrm{obs}}} \operatorname{SFR}(t) L(t) \mathrm{d} t}$,

where $\operatorname{SFR}(t)$ is the SFR as a function of time, and $L(t)$ is the luminosity emitted in the considered band per unit of formed stellar mass from the ensemble of single stellar populations of age $\left(t-t_{\mathrm{obs}}\right)$ in the galaxy (see Fig. 1). Keeping in mind the spectral evolution of a simple stellar population as a function of time (e.g., Fig. 9 of Bruzual \& Charlot 2003), it is easy to understand that $L(t)$ is a strong function of the considered photometric band. In particular, the young stellar populations represent a stronger component in the ultraviolet bands than in the optical/NIR bands and, conversely, the old stellar populations have a higher weight in the optical/NIR bands than in the ultraviolet ones.

As a consequence, for any composite stellar population (i.e., a superposition of multiple SSPs of different ages), the 


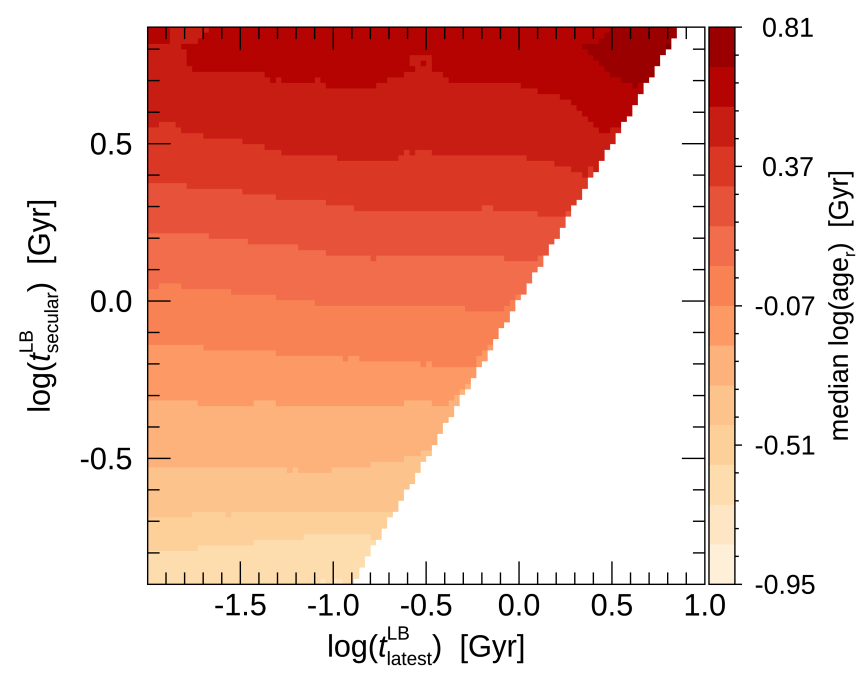

Fig. 3. Look-back time to the peak of the secular SFH ( $\left.t_{\text {secular }}^{\mathrm{LB}}\right)$ as a function of look-back time to last episode of star formation $\left(t_{\text {latest }}^{\mathrm{LB}}\right)$, colorcoded according to $r$-band light-weighted age $\left(\right.$ age $\left._{r}\right)$ for models with ages younger than the age of the Universe at redshift $z=0.55$.

light-weighted ages in different bands are expected to differ, namely in the sense of ages weighted in bluer bands being younger than those weighted in redder bands. The actual difference in the ages weighted in any two bands is determined by the characteristics of the SFH and specifically by the age spread by the different SSPs (see Sect. 2.4). Such a spread can occur even in a simple $\tau$ model, provided that $t_{\text {secular }}^{\mathrm{LB}}$ is small enough, or $\tau$ is large enough relative to $t_{\text {secular }}^{\mathrm{LB}}$ to ensure that the young and the old SSPs contribute a comparable amount of light. Similarly, when $t$ secular is large and the galaxy is in the declining phase of its $\mathrm{SFH}$, the presence of a new burst of star formation may cause a significant difference in light-weighted ages in different photometric bands.

In this work, we focus on exploiting this effect to trace the coexistence of widely diverse stellar populations and provide an essential but significant characterization of the SFH. We elect the $r$ band as the reference "red" band, and the $u$ band as the reference "blue" band. Thus, we define $r$-band light-weighted ages $\left(\right.$ age $\left._{r}\right), u$-band light-weighted ages $\left(\operatorname{age}_{u}\right)$, and their difference $\Delta($ age $)=$ age $_{r}-$ age $_{u}$, respectively. These choices are justified by the fact that these bands are well covered in the rest-frame of intermediate redshift galaxies that will be observed using newgeneration spectrographs like WEAVE or 4MOST (de Jong et al. 2019). We note that, in principle, estimates of age weighted on a given band can be obtained irrespective of that band being observed or not, because that age is a model-derived quantity, which can be extrapolated. However, it is clear that much-betterconstrained values can be obtained if that band is covered by spectroscopic observations.

We also note that we could have used the mass-weighted age (i.e., the actual first moment of the $\mathrm{SFH}$, defined by replacing $L(t)$ with 1 in Eq. (2)), because it is maximally sensitive to the age of the bulk of the stars (see also discussion in Zibetti et al. 2017). Nevertheless, its determination is much more modeldependent than for any light-weighted quantity, as the oldest stellar populations easily leave the spectrum unaffected, almost irrespective of their mass contribution. On the contrary, age ${ }_{r}$ is by definition much better constrained by the observed spectrum, yet it is a reasonably good proxy to the mass-weighted age, as we demonstrate in the following sections.

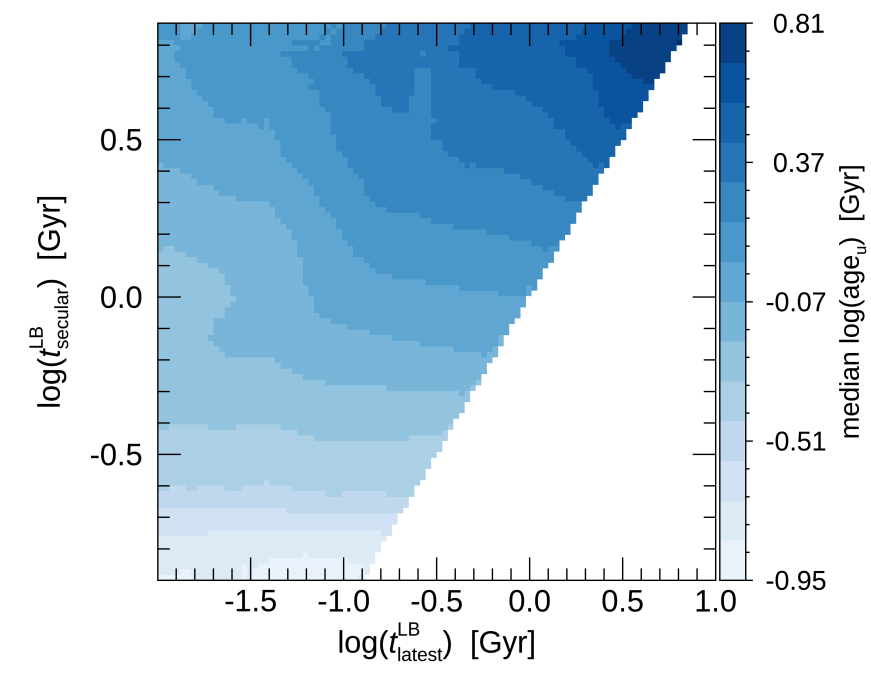

Fig. 4. Look-back time to the peak of the secular SFH $\left(t_{\text {secular }}^{\mathrm{LB}}\right)$ as a function of look-back time to the last episode of star formation $\left(t_{\text {latest }}^{\mathrm{LB}}\right)$, color-coded according to $u$-band light-weighted age $\left(\mathrm{age}_{u}\right)$ for models with ages smaller than the age of the Universe at redshift $z=0.55$.

\section{3. age $_{r}$ and $\mathrm{age}_{u}$ diagnostics}

In order to better illustrate the physical meaning of $\mathrm{age}_{r}$ and age $_{u}$ in the case of realistic SFHs, we revert to the model formalism developed in the previous sections. We stress, however, that the conclusions are not specific to this formalism but can be extended to more complex or even completely stochastic SFHs. We focus on redshift $z=0.55$, which corresponds to the median redshift where the bulk of StePS galaxies are going to be observed. We therefore select the subset of model galaxies that have younger ages than the age of the Universe at this redshift.

In Fig. 3 we highlight the role of age $_{r}$ in tracing the evolution of the bulk of the stellar population using the parameter plane of $t_{\text {secular }}^{\mathrm{LB}}$ (the look-back time when the peak of the secular SFH occurs) and $t_{\text {latest }}^{\mathrm{LB}}$ (the look-back time of the latest burst). Galaxies evolving secularly according to Fig. 1 are located along the diagonal $\left(t_{\text {secular }}^{\mathrm{LB}}=t_{\text {latest }}^{\mathrm{LB}}\right)$ and display a clear regular trend: age $_{r}$ smoothly increases from younger galaxies (smaller values of $t_{\text {secular }}^{\mathrm{LB}}$ ) to older galaxies (higher $t_{\mathrm{se}}^{\mathrm{LB}}$ values). Galaxies that experience a secondary burst in their SFHs (see Fig. 2) are offset from the diagonal position, but no significant gradient appears moving from smaller to higher $t_{\text {latest }}^{\mathrm{LB}}$, making age $r$ largely unaffected by the presence and timing of the most recent secondary burst. In other words, the value of age $e_{r}$ retains the memory of the epoch of formation of the bulk of the stellar mass, being mostly insensitive to recently formed younger stellar populations.

The picture changes with age $e_{u}$, as shown in Fig. 4, which highlights the different behavior of $\mathrm{age}_{u}$ relative to $\mathrm{age}_{r}$ in the same plane. For $\log \left(t_{\text {secular }}^{\mathrm{LB}} / \mathrm{Gyr}\right) \gtrsim-0.3$, age $_{u}$ appears to depend on both parameters and in particular to be very sensitive to the youngest bursts $\left(\log \left(t_{\text {latest }}^{\mathrm{LB}} / \mathrm{Gyr}\right) \lesssim-0.5\right)$, a sensitivity that increases as $t_{\text {latest }}^{\mathrm{LB}}$ decreases. It is worth noting that even for secularly evolving galaxies, and more clearly for bursty SFHs, there is a difference between $u$ - and $r$-band light-weighted ages at fixed $t_{\text {secular }}^{\mathrm{LB}}$, with age $_{u}$ appearing to always indicate slightly younger ages than $\operatorname{age}_{r}$, as expected.

\section{4. $\Delta$ (age) diagnostic}

We compare the different behavior of age $_{r}$ and $\mathrm{age}_{u}$ in the $\left(t_{\text {secular }}^{\mathrm{LB}}, t_{\text {latest }}^{\mathrm{LB}}\right)$ plane, exploring in Fig. 5 how $\Delta($ age $)$ may be 


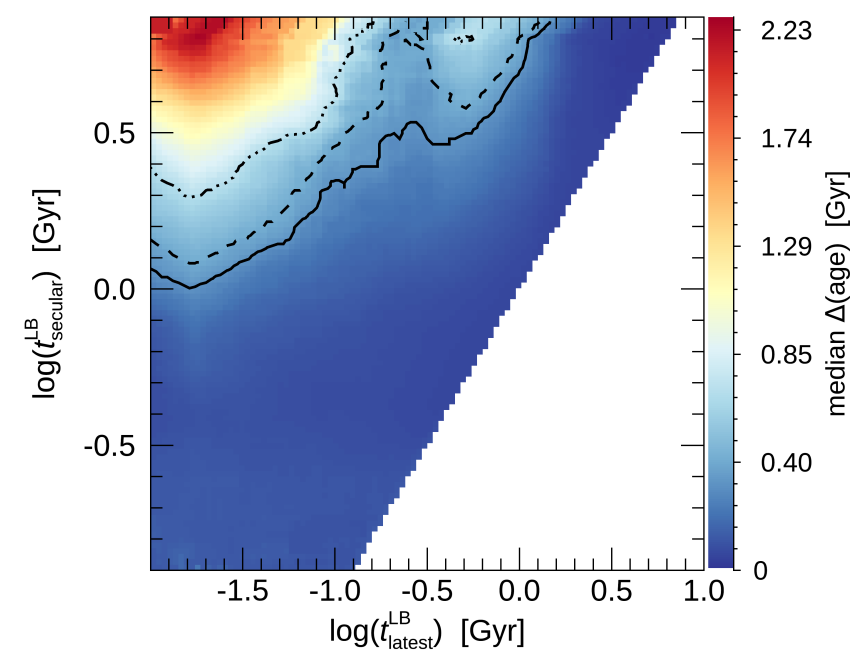

Fig. 5. Look-back time to the peak of the secular SFH $\left(t_{\text {secular }}^{\mathrm{LB}}\right)$ as a function of look-back time to the last episode of star formation $\left(t_{\text {latest }}^{\mathrm{LB}}\right)$, color-coded according to $\Delta$ (age) for models with younger ages than the age of the Universe at redshift $z=0.55$. As discussed in Sect. 5.2.2, contour lines indicate the level for which we can reliably assess a value of $\Delta$ (age) $\neq 0$ at different $S / N_{I, \text { obs }}$. In particular, the threshold is $\Delta$ (age) $=0.7$ at $S / N_{I, \text { obs }}=10$ (black dashed-dotted line), $\Delta($ age $)=0.4$ at $S / N_{I, \text { obs }}=20$ (black dashed line), and $\Delta($ age $)=0.3$ at $S / N_{I, \text { obs }}=30$ (black solid line), respectively.

used to highlight the presence of a young stellar component superimposed on an older stellar population. In this plane, the vertical gradient suggests that a greater $\Delta$ (age) value may arise only when the bulk of the galaxy stellar population is older. More importantly, the horizontal trend implies that galaxies which experienced an additional burst event display a positive and nonnegligible value of $\Delta$ (age), with more recent bursts associated with higher $\Delta$ (age) values. Thus, the larger the time separation between the peak of the secular star formation and the latest peak of star formation, the larger $\Delta$ (age) becomes. When, on the contrary, either $t_{\text {latest }}^{\mathrm{LB}}$ is quite large (greater than $1.5 \mathrm{Gyr}$ ) or the age of the peak of the secular SFH is quite small (lower than $1 \mathrm{Gyr}$ ), the value of $\Delta$ (age) becomes negligible. These are the cases where the youngest population is not young enough to leave an imprint on the value of $\Delta$ (age) or the bulk of the galaxy population is still young, so that no $\Delta$ (age) is appreciable. It is worth noting that a non-zero value of $\Delta$ (age) arises also if the galaxy is still experiencing the peak of its SFH and $\tau$ is long enough to allow the presence of a significant fraction of young stars within the galaxy, as already mentioned in Sect. 2.2.

One may expect that the mass fraction involved in the last burst event $\left(f_{\text {latest }}\right)$ plays a significant role in affecting the value of $\Delta$ (age). This possibility is explored in Fig. 6, where the plane $f_{\text {latest }}$ versus $t_{\text {latest }}^{\mathrm{LB}}$ is color-coded according to the value of $\Delta$ (age). While no trend appears in $f_{\text {latest }}$, a gradient is seen from higher to smaller values of $t_{\text {latest }}^{\mathrm{LB}}$, highlighting the fact that the time elapsed since the latest burst event is more important in affecting $\Delta$ (age) values than the fraction of mass involved in it. This trend also holds when considering higher mass fractions involved in the last burst event since no systematic variation appears in the last bin (upper part of Fig. 6), which corresponds to $\sim 10 \%$ of galaxies having $0.1<f_{\text {latest }}<0.5$. Therefore, we conclude that a significant value of $\Delta$ (age) is present when a recent burst of star formation unsettles the undisturbed secular evolution of galaxies, almost irrespective of the mass fraction involved. This lack of a clear dependence on mass fraction can be understood by recalling that we are considering light-weighted

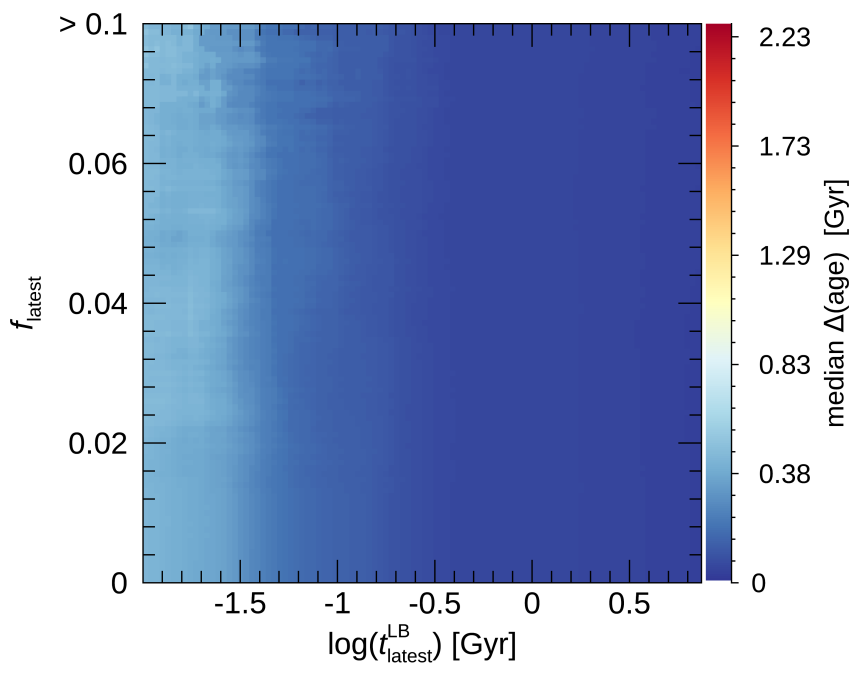

Fig. 6. Mass fraction $\left(f_{\text {latest }}\right)$ involved in last burst event as a function of the look-back time to last episode of star formation $\left(t_{\text {latest }}^{\mathrm{LB}}\right)$, colorcoded according to $\Delta$ (age) for models with ages smaller than the age of the Universe at redshift $z=0.55$. $f_{\text {latest }}>0.1$ stands for $\sim 10 \%$ of models having a large fraction of mass involved in the burst, as detailed in Sect. 2.4.

ages and that the luminosity is a strong function of age itself. What is most relevant in determining $\Delta$ (age), besides the difference in age of the two components, is the fraction of luminosity (not of mass) in the two components. If the luminosity in one of them is negligible relative to the other, no age difference emerges. Moreover, for any given mass ratio, the relative luminosity difference in the two components is determined by their absolute age in addition to their age difference. These three factors (mass ratio, absolute age, and age difference) all play degenerate roles in determining $\Delta$ (age). For these reasons we conclude that $\Delta$ (age) is a powerful probe of age spread in stellar populations, and specifically of young bursts in old galaxies, yet its amplitude cannot be directly used to estimate the mass fraction and/or the precise age of the burst.

We further checked and confirmed the absence of any systematic dependence on metallicity of $\Delta$ (age) values, splitting models into subsolar, solar, and supersolar bins. This remarkable result enables the use of this new diagnostic, disregarding the well-known degeneracy between age and metallicity, and marginalizing over the latter.

Finally, even if we are proposing an interpretation of $\mathrm{age}_{r}$ and $\Delta$ (age) based on the specific SFH of our library models, we would like to stress that these parameters per se are very general and of simple physical interpretation even when changing the specific parametric form chosen to define galaxy secular evolution. Their values may easily be used as simple heuristic information to be correlated with the variety of other intrinsic galaxy properties, such as morphology, mass, size, and environment.

\section{Classical view from optical and ultraviolet spectral indices}

In Sect. 2.4, we show that the value of $\Delta$ (age) is directly related to the presence of younger stellar components that coexist with older ones. In the literature, many attempts to describe and reconstruct the complexity of the SFHs of galaxies on the basis of the combination of spectral indices can be found (Thomas et al. 2005; Jørgensen \& Chiboucas 2013; Lonoce et al. 2014). 

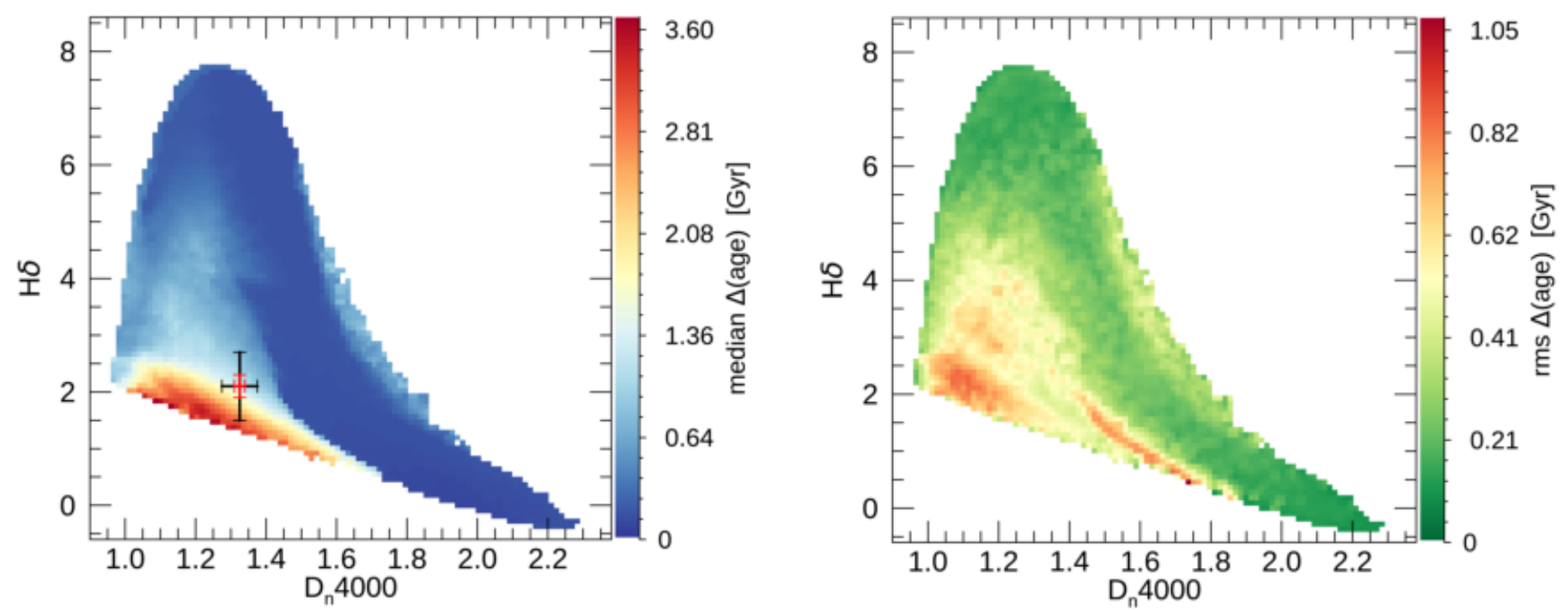

Fig. 7. $\left(\mathrm{D}_{\mathrm{n}} 4000, \mathrm{H} \delta\right)$ diagnostics color-coded according to the difference between $r$ - and $u$-band light-weighted ages for all models of the StePSlike spectral library (left panel), and color-coded according to rms in $\Delta$ (age) (right panel). We superimposed typical $1 \sigma$ average uncertainties in measuring spectral indices in this plane at $S / N=10$ (black error bars) and $S / N=30$ (red error bars), respectively.
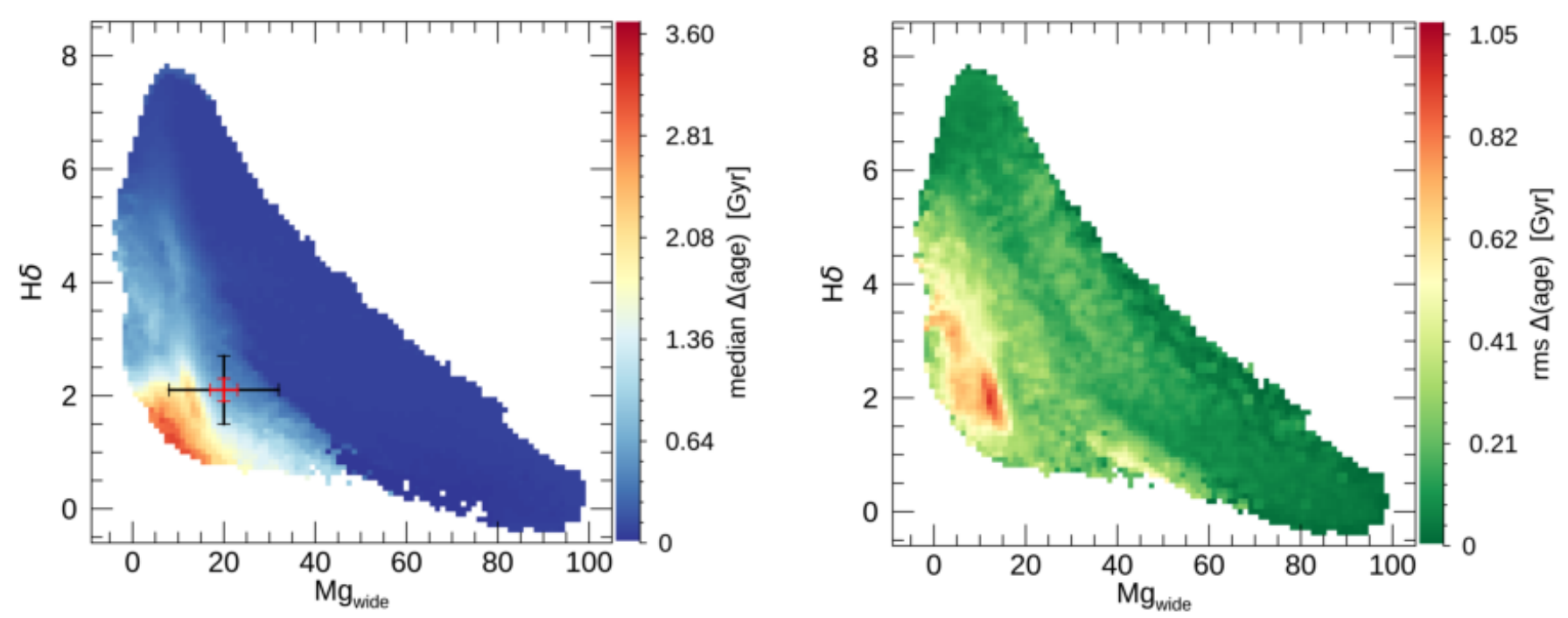

Fig. 8. $\left(\mathrm{Mg}_{\text {wide }}, \mathrm{H} \delta\right)$ diagnostics color-coded according to the difference between $r$ - and $u$-band light-weighted ages for all models of the StePSlike spectral library (left panel), and color-coded according to rms in $\Delta$ (age) (right panel). We superimposed typical $1 \sigma$ average uncertainties in measuring spectral indices in this plane at $S / N=10$ (black error bars) and $S / N=30$ (red error bars), respectively.

In particular, the $\left(\mathrm{D}_{\mathrm{n}} 4000, \mathrm{H} \delta\right)$ diagnostic has been widely used to infer the recent star formation in galaxies both in the local Universe (Kauffmann et al. 2003) and at intermediate (Wu et al. 2018) or high redshift (Onodera et al. 2012).

More recently, ultraviolet spectral features have been used to derive the physical properties of the stellar content of galaxies (Fanelli et al. 1992; Daddi et al. 2005; Maraston et al. 2009; Vazdekis et al. 2016). In particular, the $\mathrm{Mg}_{\text {wide }}$ index has been demonstrated to be sensitive to the presence of stars older than $0.5 \mathrm{Gyr}$ and it can be used as an alternative to the $\mathrm{D}_{\mathrm{n}} 4000$ index as a signature of the ages of the old stellar components in galaxies (Daddi et al. 2005).

In the following, we therefore explore the possibility of inferring $\Delta$ (age) using both classical spectral indices in the optical wavelength range (e.g., $\mathrm{D}_{\mathrm{n}} 4000$ and $\mathrm{H} \delta$ ) and combining spectral indices in the ultraviolet and optical wavelength range (e.g., $\mathrm{Mg}_{\text {wide }}$ and $\mathrm{H} \delta$ ).
In Fig. 7 (left panel), we present the classic $\left(\mathrm{D}_{\mathrm{n}} 4000, \mathrm{H} \delta\right)$ diagnostic as derived for the parent library models, color-coded according to the difference between the $r$ - and $u$-band lightweighted ages. Galaxies that present a negligible amplitude of $\Delta$ (age) $\sim 0$ are arranged in the bell-shaped sequence characteristic of the time evolution of SSPs (e.g., Fig. 3 of Kauffmann et al. 2003), that is, generations of stars with negligible spread in age. The sequence is defined starting with the youngest SSPs at the lowest $\mathrm{D}_{\mathrm{n}} 4000$ and intermediate $\mathrm{H} \delta$, then moving to the 1-2 Gyr-old SSPs peaking at the maximum values of $\mathrm{H} \delta$ at intermediate and low $\mathrm{D}_{\mathrm{n}} 4000$, and finally to the oldest SSPs at the highest values of $D_{n} 4000$ and the lowest values of $H \delta$. The region below the bell shape is characterized by increasing values of $\Delta$ (age), moving from the top to the bottom. This can be understood in terms of mixing old and young stellar populations, which are both characterized by low $\mathrm{H} \delta$ and whose high and low values of $D_{n} 4000$, respectively, average out to an intermediate 
value for the break. In particular, we note the extreme region of $\mathrm{H} \delta \lesssim 2.5$ and intermediate $\mathrm{D}_{\mathrm{n}} 4000$, which is characterized by $\Delta$ (age) $\gtrsim 2$ Gyr. Models with these properties are the result of a tiny fraction $(\lesssim 0.1 \%)$ of young stars (age $\lesssim 10^{8} \mathrm{yr}$ ) "frosted" on the top of a stellar population of several gigayears in age.

However, in order to fully appreciate the usefulness of such a plot as a tool to estimate $\Delta$ (age), we need to consider the intrinsic scatter of $\Delta$ (age) in the same plane (Fig. 7, right panel), as well as the typical observational errors in measuring spectral indices (Fig. 7, left panel). The scatter reveals the intrinsic degeneracy between the values of the spectral indices and the physical quantity $\Delta$ (age), and makes this diagnostic a less straightforward proxy for $\Delta$ (age), even if it ideally provides a relatively good characterization of the difference of light-weighted ages. Considering typical uncertainties on spectral indices, the overall picture inevitably blurs (see Fig. 7 and Sect. 5.1). This makes it almost impossible to infer a reliable value of $\Delta$ (age) using only the information provided by two spectral indices, especially in the region of the plane of higher $\Delta$ (age) values.

Since the ultraviolet wavelength flux correlates well with the presence of young stellar populations, one may expect that moving to ultraviolet spectral indices may help to alleviate the intrinsic scatter shown in Fig. 7. Therefore, we explore in Fig. 8 (left panel) the possibility of using the diagnostic plane $\left(\mathrm{Mg}_{\text {wide }}\right.$, $\mathrm{H} \delta$ ) that takes advantage of the wide magnesium absorption feature in the bluer part of the spectrum, to trace the presence of recent events of star formation. This feature becomes available for galaxies at redshift $z \gtrsim 0.55$ in spectrographs whose coverage in wavelength starts at $3800 \AA$ (as foreseen for WEAVE and 4MOST; see also Sect. 5.1). As in Fig. 7 (left panel), we can identify a continuum sequence followed by coeval stellar populations, from high values of the $\mathrm{Mg}_{\text {wide }}$ index combined with low values of $\mathrm{H} \delta$, up to low values of $\mathrm{Mg}_{\text {wide }}$ combined with high values of $\mathrm{H} \delta$. As in the classical diagram, we can identify a region where the $\mathrm{Mg}_{\text {wide }}$ index assumes values lower than 50, which indicate stellar populations younger than a few gigayears, and $\mathrm{H} \delta$ values lower than 4-5, which reveal the presence of very young components (i.e., ages lower than $0.1 \mathrm{Gyr}$ ). The two indices in this region point toward the presence of stellar populations of different ages, and indeed they correspond to the region where $\Delta$ (age) is larger than $0.4-0.5 \mathrm{Gyr}$ on average. In our parent library, it appears that the use of ultraviolet indices in the new $\left(\mathrm{Mg}_{\text {wide }}, \mathrm{H} \delta\right)$ diagnostic reduces the degree of intrinsic uncertainty in the transition region of $\Delta$ (age) $\sim 1$ Gyr (see Fig. 8, right panel). On the other hand, as stated before and as shown in the following sections, since we have to consider the nonzero (and usually non-negligible, even at high $\mathrm{S} / \mathrm{N}$ ) uncertainties in measuring spectral indices, only by taking into account the whole spectral information and dealing with a Bayesian analysis is it possible to properly retrieve and constrain the $\Delta$ (age) of galaxies.

\section{From models to simulated observations}

One of the main goals of this paper is to provide a realistic appraisal of the scientific opportunities created by data from new-generation wide-field spectrographs at four-meterclass telescopes: large samples of galaxy spectra of high resolution $(R \sim 5000)$, moderate quality $\left(S / N \gtrsim 10 \AA^{-1}\right)$, and wide wavelength coverage.

In this context, StePS can be considered the forerunner of the new observations that these spectrographs will provide. It uses the WEAVE spectrograph on the WHT in its so-called Low-
Resolution MOS mode $(R \sim 5000$, over the wavelength range of 3660-9590 $\AA$ ) to study galaxy evolution out to $z=0.7$ for a sample of approximately 25000 galaxies. Its target sample is selected using a simple magnitude criterion $\left(I_{\mathrm{AB}} \leq 20.5 \mathrm{mag}\right)$ coupled with a photometric (spectroscopic when available) redshift pre-selection $\left(z_{\text {phot }} \geq 0.3\right)$. The survey strategy foresees seven hours of observations for each target, split into 21 observing blocks of $\sim 20 \mathrm{~min}$ each, enabling us to reach $S / N \gtrsim 10 \AA^{-1}$ in $I$ band for most of the targets.

We simulate mock observed galaxy spectra using a typical StePS observational setup and the known parameters of the WEAVE spectrograph on the WHT (see also Jin et al., in prep. for a set of comprehensive simulations of the forthcoming WEAVE data), and use this sample to explore the physical information that can be extracted from it.

Even though we use StePS as the blueprint for our simulations, the flexibility of our method and analysis is such that it can be easily extended or modified to closely reproduce data from other upcoming facilities (e.g., 4MOST at VISTA or PFS at SUBARU, to mention similar projects in advanced stages of completion; Takada et al. 2014).

\subsection{StePS-like simulated observations: the ingredients}

As already anticipated in Sect. 2.1, we make use of a representative, randomly chosen subsample of 12500 rest-frame templates, stemming from the full parent library of 500000 model spectra, to produce mock StePS-like observations, that is, spectra that closely mimic forthcoming WEAVE-StePS data. For each considered redshift $(z=[0.3,0.55,0.7])$, we make a selection of models from the chunk, which are required to have (i) $t_{\text {obs }}$ not exceeding the age of the Universe at that redshift, (ii) $g$-band effective attenuation $A_{g} \equiv M_{g \text {,emerging }}-M_{g \text {,emitted }}<2$ mag, (iii) $r$-band light-weighted mean metallicity $0.1<Z / Z_{\odot}<2$. Both metallicity and attenuation values are appropriate for the typical mass range of galaxies observed in surveys with bright selection magnitude limit $\left(I_{\mathrm{AB}}<20.5 \mathrm{mag}\right.$, see Zahid et al. 2014). With this selection, we obtain 4848 models at $z=0.3,4300$ models at $z=0.55$, and 3967 models at $z=0.7$, respectively. It is worth noting that this subsample library is fully representative of the comparison library, ensuring no bias in any physical property (in particular in SFHs), and is sufficient in size to enable an exploration of the whole parameter space of galaxy physical properties with statistically significant numbers.

Two ingredients are needed in order to reproduce mock StePS-like observations starting from these model spectra:

(a) $\mathrm{RF}_{\lambda, \text { obs }}$ : the throughput of the combined atmospheric transmission, optics of the WHT and WEAVE spectrograph, including also the gaps due to the presence of inter-CCD spacing in both blue and red spectrograph arms;

(b) $N_{\lambda, \text { obs }}$ : the contribution to the noise for all our mock StePSlike spectra due to the expected Poisson noise from sky continuum background and the detector noise of WEAVE CCDs. The value of $\mathrm{RF}_{\lambda, \mathrm{obs}}$ is obtained from Dalton et al. (2016) for the typical atmospheric transmission values and for combined WEAVE+WHT throughput. The sky Poisson noise is computed assuming a typical sky continuum spectrum as provided in ESO Exposure Time Calculators and a dark-sky surface brightness magnitude of $V \sim 22.0 \mathrm{mag} \operatorname{arcsec}{ }^{-2}$, which can be considered typical for sky brightness of dark nights in La Palma (Benn \& Ellison 1998). Using the function $\mathrm{RF}_{\lambda \text {,obs }}$ described in (a) and the footprint on sky of the WEAVE fibers ( 1.3 arcsec as defined by diameter of fiber core), we obtain the expected sky background Poisson noise in counts $\mathrm{px}^{-1}$ on the CCDs (gain $=1$ ). We highlight 


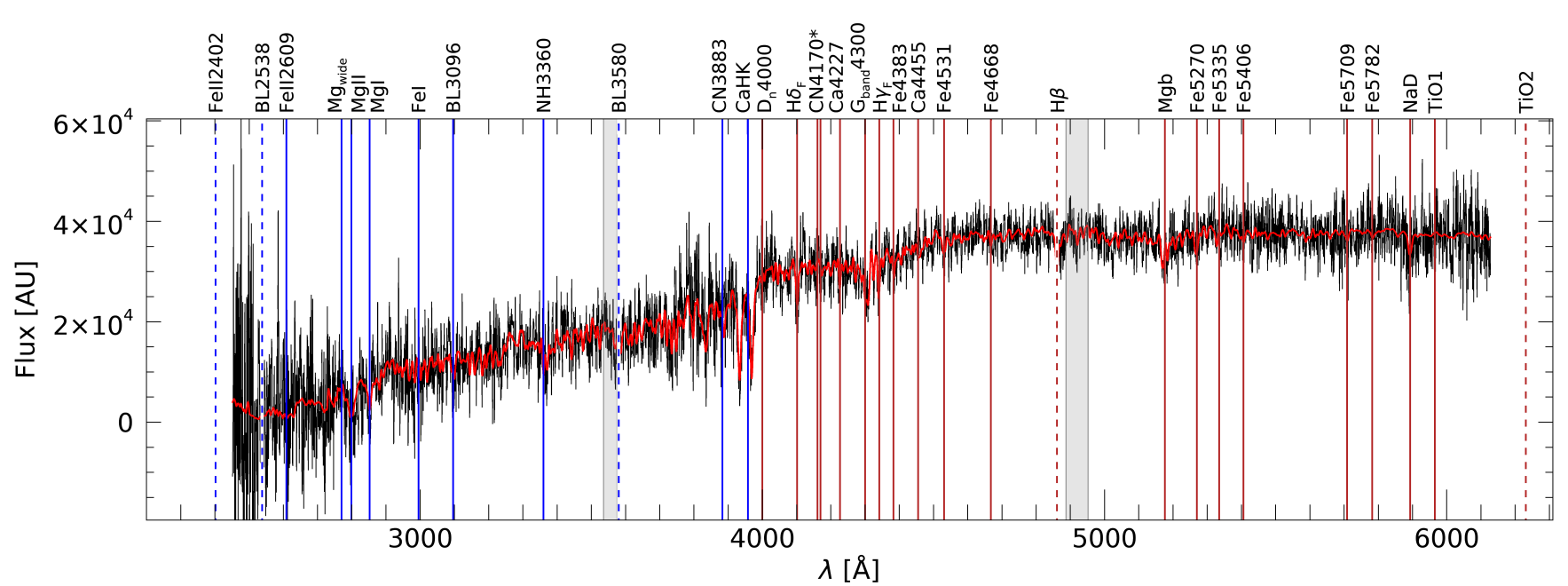

Fig. 9. Example of a rest-frame template (red curve) and observed spectrum (black curve) that mimic the StePS observation of a galaxy at $z=0.55$ and $S / N_{\mathrm{I}, \mathrm{obs}}=10$. The central rest-frame wavelengths of ultraviolet spectral indices are marked with blue solid lines, while those of optical spectral indices are marked with red solid lines. $\mathrm{CN} 4170$ and $\mathrm{CN}_{2}$ features share similar positions $\left(^{*}\right)$ in the plot. Gray vertical bands represent gaps in the WEAVE spectrograph. It is worth noting that FeII2402, BL2538, and TiO2 indices are outside the considered spectral range, while BL3580 and $\mathrm{H} \beta$ features fall in the gap of the $\mathrm{CCD}$ at this particular redshift (dashed lines).

the fact that in our estimate of sky Poisson noise we neglect the contribution of sky emission lines, as the relatively high resolution used in StePS $(R \sim 5000)$ is such that the pixels affected by the presence of such lines can be easily masked in the analysis.

The contribution of the WEAVE detectors to noise is mainly due to read-out noise, which from WEAVE specifications (Dalton et al. 2016) we assume to amount to $\sim 2.5 \mathrm{e}^{-} \mathrm{px}^{-1}$. We estimate its actual value in counts for our spectra by considering an exposure time of $21 \times 20 \mathrm{~min}, \sim 7 \mathrm{~h}$, and a typical spatial diameter of a fiber on the CCDs of $\sim 4.5 \mathrm{px}$, corresponding to an expected integration window for profile-weighted extraction of approx $1.5 \times F W H M$ px. We neglected the contribution of thermal noise due to dark current, as the exposure time for single observing blocks in StePS will be short ( $20 \mathrm{~min})$ and Dark Current for WEAVE detectors is relatively low $\left(\leq 0.1 \mathrm{e}^{-} \mathrm{h}^{-1}\right)$.

While the first ingredient, $\mathrm{RF}_{\lambda, \mathrm{obs}}$, is used to convert all our model spectra fluxes to expected counts on the CCDs, thus defining (in arbitrary units) the shape of the spectra as observed, the second one, $N_{\lambda, \mathrm{obs}}$, provides a realistic wavelength-dependent noise contribution in counts that is equal for all our sources. This noise is added in quadrature to the specific Poisson noise for each template to generate mock StePS-like observed spectra. Its value is used to anchor, depending on the desired $\mathrm{S} / \mathrm{N}$ in $I$-band, the actual value of counts (and therefore the corresponding Poisson noise contribution) of the observed mock StePS-like spectra. These two ingredients are obviously specific to WEAVE+WHT, but can easily be changed to reproduce different spectrographs and telescopes characteristics.

\subsection{StePS-like simulated observations: the recipe}

In this section we detail the procedure to move from our representative subsample of rest-frame templates to mock StePS-like observed spectra at three different redshifts $z=[0.3,0.55,0.7]$, and three $S / N_{I, \text { obs }}=[10,20,30] \AA^{-1}$. In sequence, we perform the following operations.

(1) Each model template $\mathcal{T}_{\lambda}$ is redshifted to each of the three desired redshift values $z=[0.3,0.55,0.7]$, rebinned to the observed pixel size equal to $1 \AA$, and finally trimmed to fit the WEAVE spectral range, becoming $\mathcal{T}_{\lambda \text {,obs }}$.
(2) Each template $\mathcal{T}_{\lambda \text {,obs }}$ is then multiplied by the function $\mathrm{RF}_{\lambda, \text { obs }}$, in order to retrieve the correct shape, in arbitrary units, of observed counts on the CCDs, becoming $\mathcal{T}_{\lambda \text {,obs }}$ [counts $\AA^{-1}$ ].

(3) For each template, we then compute the value $S_{I, \mathrm{obs}}$, that is, the mean number of counts per $\AA^{-1}$ needed to get the desired $I$-band $S / N_{I, \text { obs }}=[10,20,30]$. We use the function $N_{\lambda, \text { obs }}$ to derive $N_{I, \mathrm{obs}}$, that is, the expected sky+CCD noise contribution in counts $\AA^{-1}$ in $I$-band. For each $S / N_{I, \text { obs }}$ defined above, $S_{I, \text { obs }}$ can then be simply obtained using the formula:

$S / N_{I, \mathrm{obs}}=\frac{S_{\mathrm{I}, \mathrm{obs}}}{\sqrt{S_{I, \mathrm{obs}}+N_{I, \mathrm{obs}}^{2}}}$.

(4) The value of $S_{I, \mathrm{obs}}$ is then used to normalize each template $\mathrm{T}_{\lambda, \text { obs }}$ [counts $\AA^{-1}$ ] to obtain spectra of $S / N_{I, \mathrm{obs}}=$ $[10,20,30]$.

(5) At each wavelength, we calculate the Poisson noise contribution in counts for each normalized template and add it in quadrature to the sky+CCD noise $N_{\lambda \text {,obs }}$ contribution, to obtain the total noise budget $N_{\lambda, \mathrm{obs}}^{\mathrm{tot}}$.

(6) Each normalized template $\mathcal{T}_{\lambda \text {,obs }}$ is perturbed with a noise contribution randomly generated from Gaussians of width equal to $N_{\lambda, \mathrm{obs}}^{\text {tot }}$, obtaining observed spectra $\mathrm{S}_{\lambda, \mathrm{obs}}$.

(7) We apply back the sensitivity function to all spectra $\mathcal{S}_{\lambda \text {,obs }}$, obtaining the final spectra $\mathcal{S}_{\lambda \text {,obs }}$ back in flux units.

At the end of this procedure we then have, for each model template $\mathcal{T}_{\lambda}$, realistic mock StePS-like observed spectra $\mathcal{S}_{\lambda \text {,obs }}$ that mimic StePS observations at the three redshifts for each of the three $S / N_{I, \text { obs }}$ chosen (see Fig. 9, as an example).

The realism introduced by the wavelength dependence of $S / N_{\lambda \text {,obs }}$ enables us to take into account in our simulations the difficulties of dealing with the variety of spectral types (including redder galaxies) and the reduced efficiency of the WEAVE+WHT system in going to bluer wavelengths. Our library of mock StePS-like observed spectra can assess, in a reliable fashion, what can be obtained from spectra of different $S / N_{I, \text { obs }}$ values and spectral types at different redshifts. However, we note that with our simulations we only reproduce the random 


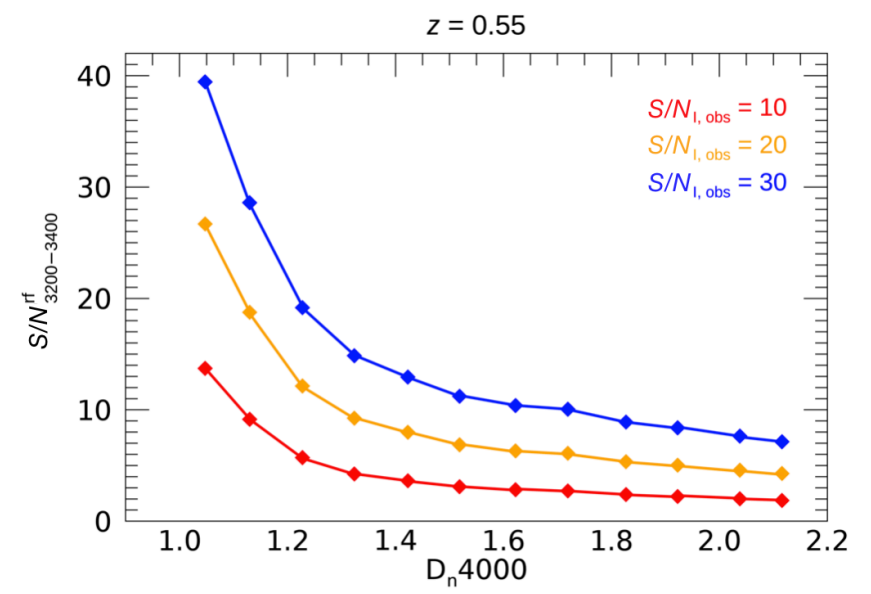

Fig. 10. Median value of $S / N_{3200-3400}^{\mathrm{RF}}$ as a function of $\mathrm{D}_{\mathrm{n}} 4000$ at fixed $z=0.55$. Different colors correspond to $S / N_{I, \mathrm{obs}}=10$ (red diamonds), $S / N_{I, \mathrm{obs}}=20$ (orange diamonds), and $S / N_{I, \mathrm{obs}}=30$ (blue diamonds).

uncertainties due to the noise in the spectra, while we do not include any subdominant systematic errors that might be present in the observed data (e.g., sky subtraction, flux calibration).

To quantify our capability to retrieve ultraviolet indices in observed spectra, in Fig. 10 we analyze $S / N_{3200-3400}^{\mathrm{RF}}$, that is the trend of $\mathrm{S} / \mathrm{N}$ as directly measured from mock observed spectra in the rest-frame window $3200-3400 \AA$ as a function of different $\mathrm{D}_{\mathrm{n}} 4000$ values for three values of $S / N_{I, \text { obs }}=[10,20,30]$ at fixed redshift $z=0.55$. Our ability to measure ultraviolet indices strongly depends not only on the $S / N_{I, \text { obs }}$ value, but also on the spectral type of the galaxies as identified by $D_{n} 4000$ values. In particular, for $D_{n} 4000<1.5$, the $S / N$ in the blue region of the spectra increases significantly, thus increasing the reliability of the analysis based on ultraviolet indices. This trend does not change when moving to redshifts $z=0.3$ and $z=0.7$.

\section{Stellar population analysis}

As we discuss in Sect. 3, a simple index-versus-index plane, even when including ultraviolet indices, cannot fully capture the complexity that is beyond the median value of $\Delta$ (age) corresponding to a specific position in such a plane. The large scatter of possible $\Delta$ (age) values and the typical uncertainties associated with measured spectral indices in the positions where the presence of differences in stellar ages becomes appreciable make it very difficult to apply such diagnostic tools to individual galaxies (see Figs. 7 and 8).

In this section we show how the ability to resolve the key absorption-line indices both in the ultraviolet and optical wavelength ranges enables us, when adopting a full Bayesian approach, to reliably obtain the value of $\mathrm{age}_{r}, \mathrm{age}_{u}$, and $\Delta$ (age). It is worth noting that the power of a full Bayesian analysis relies on the ability to retrieve for each individual galaxy the full PDF of any physical parameter chosen, while providing a good representation of the overall uncertainties. The analysis is performed at all redshifts and $S / N_{I, \text { obs }}$ defined in the previous section. However, we provide representative results obtained at $z=0.55$, the median redshift for StePS.

\subsection{Spectral indices}

We measure all spectral indices that can be observed in the WEAVE spectral range corresponding to each chosen redshift $z=[0.3,0.55,0.7]$, as listed in Table 1 . Indices defined for
Table 1. Ultraviolet and optical spectral indices.

\begin{tabular}{|c|c|c|c|c|c|}
\hline $\begin{array}{l}\text { UV index } \\
\text { (1) }\end{array}$ & $\begin{array}{c}z \\
(2)\end{array}$ & $\begin{array}{c}\text { Ref. } \\
(3)\end{array}$ & $\begin{array}{l}\text { Opt. index } \\
\text { (1) }\end{array}$ & $\begin{array}{c}z \\
(2)\end{array}$ & $\begin{array}{c}\text { Ref. } \\
(3)\end{array}$ \\
\hline FeII2402 & $>0.66$ & $\mathrm{a}$ & $\mathrm{D}_{\mathrm{n}} 4000$ & All & $\mathrm{c}$ \\
\hline BL2538 & $>0.56$ & $\mathrm{a}$ & CN4170 & All & d \\
\hline FeII2609 & $>0.48$ & $\mathrm{a}$ & $\mathrm{H} \delta_{\mathrm{F}}$ & All & d \\
\hline MgII & $>0.38$ & $\mathrm{a}$ & $\mathrm{Ca} 4227$ & All & $\mathrm{d}$ \\
\hline MgI & $>0.35$ & $\mathrm{a}$ & $\mathrm{H} \gamma_{\mathrm{F}}$ & All & $\mathrm{d}$ \\
\hline $\mathrm{Mg}_{\text {wide }}$ & $>0.54$ & $\mathrm{a}$ & $G_{\text {band }} 4300$ & All & $\mathrm{d}$ \\
\hline FeI & $>0.31$ & $\mathrm{a}$ & $\mathrm{Fe} 4383$ & All & $\mathrm{d}$ \\
\hline BL3096 & $>0.25$ & $\mathrm{a}$ & $\mathrm{Ca} 4455$ & All & d \\
\hline NH3360 & $>0.14$ & $\mathrm{a}$ & $\mathrm{Fe} 4531$ & All & $\mathrm{d}$ \\
\hline BL3580 & $>0.09$ & $\mathrm{a}$ & $\mathrm{Fe} 4668$ & All & $\mathrm{d}$ \\
\hline CN3883 & $>0.01$ & $\mathrm{a}$ & $\mathrm{H} \beta$ & All & $\mathrm{d}$ \\
\hline \multirow[t]{10}{*}{$\mathrm{CaHK}$} & All & $\mathrm{b}$ & Fe5015 & All & $\mathrm{d}$ \\
\hline & & & $\mathrm{Mgb}$ & All & $\mathrm{d}$ \\
\hline & & & $\mathrm{Fe} 5270$ & $<0.79$ & $\mathrm{~d}$ \\
\hline & & & Fe5335 & $<0.77$ & $\mathrm{~d}$ \\
\hline & & & Fe5406 & $<0.75$ & $\mathrm{~d}$ \\
\hline & & & Fe5709 & $<0.66$ & d \\
\hline & & & Fe5782 & $<0.63$ & $\mathrm{~d}$ \\
\hline & & & $\mathrm{NaD}$ & $<0.60$ & d \\
\hline & & & TiO1 & $<0.56$ & $\mathrm{~d}$ \\
\hline & & & $\mathrm{TiO} 2$ & $<0.48$ & $\mathrm{~d}$ \\
\hline
\end{tabular}

Notes. (1) index name; (2) redshift range in which the index is within the spectral range of the WEAVE spectrograph $\left(z_{\max }=0.8\right)$.

References. (a) Fanelli et al. (1992), (b) Serven et al. (2005), (c) Balogh et al. (1999), (d) Worthey et al. (1994).

band-pass wavelength greater than $4000 \AA$ are considered optical indices, while those defined for band-pass wavelengths lower than $4000 \AA$ are considered ultraviolet ones. An example of measured indices for an observed spectrum at $z=0.55$ is shown in Fig. 9.

In order to estimate observational errors associated with each index, we generate 1000 random realizations of noise contribution for each template $\mathcal{T}_{\lambda, \mathrm{obs}}$, at each considered $S / N_{I, \mathrm{obs}}$ and redshift. We then measure all spectral indices for each realization and evaluate the systematic $\sigma_{\text {syst }}$ and statistical $\sigma_{\text {stat }}$ observational errors as the mean and standard deviation of the relative difference between true and measured values. To realistically estimate uncertainties on the $D_{n} 4000$ spectral index, whose window of estimate is relatively wide, we included a generous extra term $(\sim 5 \%)$ to the error budget to account for the uncertainty expected on the spectrophotometric calibration. In Fig. 11 we show the systematic deviation between true and observed values of three indices, namely, $\mathrm{Mg}_{\text {wide }}, \mathrm{D}_{\mathrm{n}} 4000$, and $\mathrm{H} \delta$, for observed spectra with $S / N_{I, \text { obs }}=10$ : even in the lowest bin of $S / N_{I, \mathrm{obs}}$, there are no systematic errors in retrieving the true value of measured indices, confirming the good accuracy of our estimates.

In order to evaluate the actual contribution of each index in retrieving light-weighted ages in different bands, we calculate their resolving power $\log \left(\delta_{i} / \sigma_{i}\right)$, as defined in Gallazzi et al. (2005). In this definition, $\sigma_{i}$ is the statistical observational uncertainty associated to a particular index $\left(\sigma_{i}=\sigma_{\text {stat }}\right.$, where $i$ runs on different indices), while $\delta_{i}$ (with $i$ running on different indices) is the dynamical range of each index, which corresponds to the 5-95\% percentile range of the distribution of index strengths for all observed galaxies at a given redshift. A resolving power of zero or less indicates that the measurement 


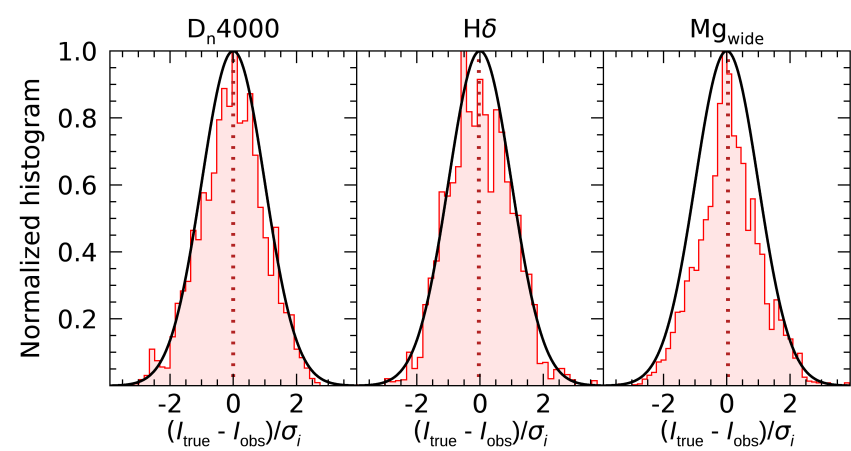

Fig. 11. Distribution of systematic errors at $z=0.55$ and $S / N_{I, \mathrm{obs}}=10$ for $\mathrm{D}_{\mathrm{n}} 4000, \mathrm{H} \delta$, and $\mathrm{Mg}_{\text {wide }}$, respectively. The red dotted line corresponds to the median value of each distribution. For reference, a solid black line shows a Gaussian distribution with unit standard deviation.

error is such that the index is broadly consistent with the full range of models, meaning that little or no information is provided. On the contrary, $\log \left(\delta_{i} / \sigma_{i}\right) \gg 0$ implies that we are able to locate the value of the index very precisely within its allowed range, and therefore we can obtain significant information. To ensure a more refined characterization of the resolving power of each spectral index measurement, for indices other than $D_{n} 4000$, we consider the dynamical range $\delta_{i}$ at a fixed value of $\mathrm{D}_{\mathrm{n}} 4000$ equal to that of the galaxy, whereby $D_{n} 4000$ is used as a proxy of galaxy spectral type. Thus, we calculate a resolving power $\log \left(\delta_{i} / \sigma_{i}\right)$ that depends on the spectral type of observed galaxies. In Fig. 12 we show the resolving power of three indices, namely, $\mathrm{Mg}_{\text {wide }}, \mathrm{D}_{\mathrm{n}} 4000$, and $\mathrm{H} \delta$, for observed spectra with $S / N_{I, \text { obs }}=10$ and $S / N_{I, \text { obs }}=30$, while an example of the actual dynamical range of $\mathrm{H} \delta\left(\delta_{\mathrm{H} \delta}\right)$ as a function of $\mathrm{D}_{\mathrm{n}} 4000$ can be obtained from Fig. 7, where clearly the dynamical range for $\mathrm{H} \delta$ is large $\left(\delta_{\mathrm{H} \delta} \sim[2,8]\right)$ at lower values of $\mathrm{D}_{\mathrm{n}} 4000$, and smaller $\left(\delta_{\mathrm{H} \delta} \sim[0,1]\right)$ at higher values of $\mathrm{D}_{\mathrm{n}} 4000$.

As expected, the resolving power of different spectral indices has an important dependence on the galaxy spectral type. Moreover, since red (blue) galaxies have a lower (higher) $S / N_{3200-3400}^{\mathrm{RF}}$, taking this dependence into account is the only way to robustly estimate the actual contribution of each measured index to our Bayesian analysis, particularly in the bluer region of the spectrum.

The expected performances of StePS are remarkable, as even at the lowest $S / N_{I, \text { obs }}$ the bluer indices are retrieved with a precision that delivers a resolving power $\log \left(\delta_{i} / \sigma_{i}\right)>0$ for most galaxies. In Fig. 12 this is shown for the index $\mathrm{Mg}_{\text {wide }}$, where $\log \left(\delta_{\mathrm{Mg}_{\text {wide }}} / \sigma_{\mathrm{Mg}_{\text {wide }}}\right)>0$ in approximately $70 \%$ of the realizations even at $S / N_{I \text {,obs }} \sim 10$. This percentage increases to almost $100 \%$ at higher values of $S / N_{I, \text { obs }}$. The same trend roughly holds for most of the ultraviolet indices explored.

\subsection{Bayesian inference}

Bayesian statistics provides a powerful framework for inferring the intrinsic properties of galaxies. As a consequence of Bayes' theorem, the probability that a certain physical parameter $\Theta$ is described by observed data $\theta$ is given by the posterior PDF:

$p(\Theta \mid \theta)=\frac{p(\Theta) p(\theta \mid \Theta)}{p(\theta)}$,

where $p(\Theta)$ is the prior distribution on $\Theta, p(\theta \mid \Theta)$ is the likelihood function, and $p(\theta)$ is a normalization factor independent of the assumed model. Our application of the Bayesian approach relies

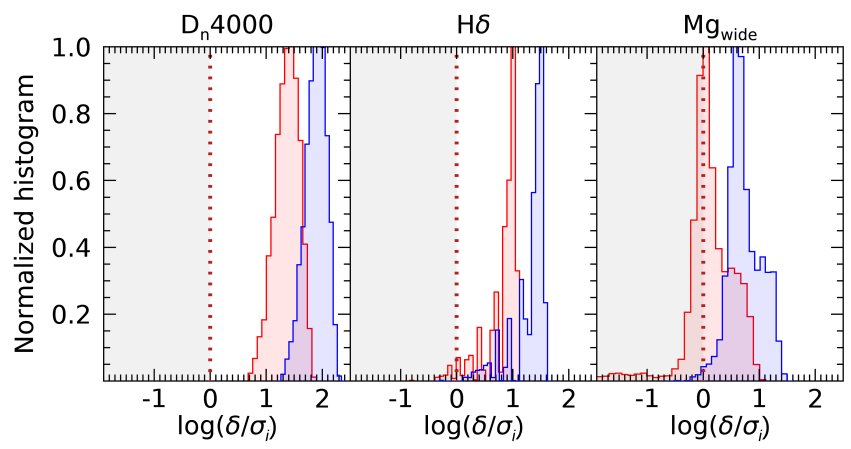

Fig. 12. Distribution of the resolving power $\log \left(\delta_{i} / \sigma_{i}\right)$ at $z=0.55$ for $\mathrm{D}_{\mathrm{n}} 4000, \mathrm{H} \delta$, and $\mathrm{Mg}_{\text {wide }}$, respectively. Red histograms correspond to $S / N_{I, \text { obs }}=10$, while blue histograms stand for $S / N_{I, \text { obs }}=30$. The red dotted line separates the region where $\log \left(\delta_{i} / \sigma_{i}\right)<0$ (in gray) from the one where $\log \left(\delta_{i} / \sigma_{i}\right)>0$ (in white).

on the comparison between a set of observable quantities consistently measured in the data (real or mock) and those provided by the models of the reference library (see Sect. 2.1). In order to keep our mock data and the reference library fully independent, the latter is selected from the complement to the chunk in the parent library. We exclude from the reference library those models that have $t_{\mathrm{obs}}$ exceeding the age of the Universe at the considered redshift by more than $1 \mathrm{Gyr}$. We allow this buffer of unphysical ages (older than the age of the Universe at the redshift considered) in order to avoid skewing the PDF of age values by cutting the old wing.

Assuming errors normally distributed with a known correlation matrix, the likelihood is proportional to the chi-square statistic

$\chi^{2}(\theta)=\sum_{i=1}^{N}\left(\frac{I_{i}^{\mathrm{obs}}-I_{i}^{\mathrm{mod}}}{\sigma_{i}}\right)^{2}$,

where $i$ runs over all observed spectral indices, $I_{i}^{\text {obs }}$ and $I_{i}^{\bmod }$ are the values of $i$ th spectral index in the observed and model spectra, respectively, and $\sigma_{i}$ is the observational error of $i$ th spectral index, computed as described in Sect. 5.1. Thus, the likelihood of a given set of observed spectral indices $\theta$ being described by a spectrum with intrinsic properties $\Theta$ is given by

$p(\theta \mid \Theta) \propto \mathrm{e}^{-\chi(\theta) / 2}$.

The Bayesian approach allows us to marginalize over different parameters, focusing on relevant physical quantities instead of the complex SFH of each galaxy (Kauffmann et al. 2003; Gallazzi et al. 2005; Zibetti et al. 2017). The PDF of each parameter is obtained by the distribution of the likelihood of all the models in the reference spectral library in that parameter space. We focus on the PDFs of three physical quantities:

- age $_{r}$, i.e., $r$-band light-weighted age;

- age $_{u}$, i.e., $u$-band light-weighted age;

- $\Delta$ (age) $=$ age $_{r}-$ age $_{u}$.

The fiducial value of each parameter is estimated as the marginalized median value of the PDF, while errors $\sigma_{\text {obs }}$ are evaluated as half of the 68 per cent confidence interval, which corresponds to the 16-84 per cent percentile range of the PDF, and is equivalent to $\pm 1 \sigma$ for a Gaussian distribution.

\subsubsection{Results: estimates of age $\mathrm{e}_{r}$ and $\mathrm{age}_{u}$}

We use the method described in Sect. 5.2 to retrieve the PDF of age $_{r}$ and age $_{u}$ of our mock observed galaxies and explore the 


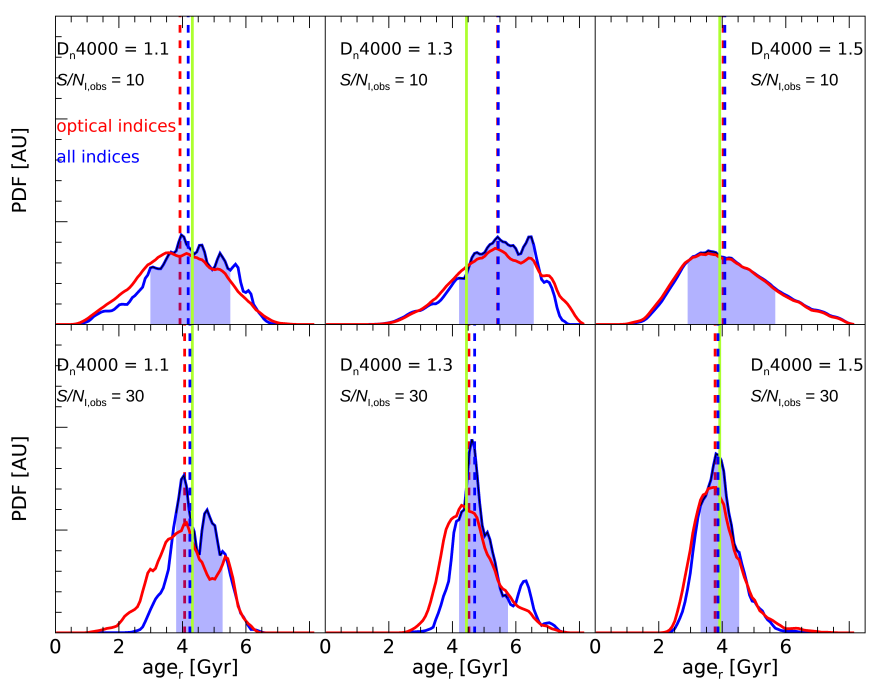

Fig. 13. Examples of PDFs of age ${ }_{r}$ of observed galaxies at $z=0.55$; different values of $\mathrm{D}_{\mathrm{n}} 4000=[1.1,1.3,1.5]$ (from left to right), and $S / N_{I, \mathrm{obs}}=10$ (upper panels) and $S / N_{I, \mathrm{obs}}=30$ (bottom panels) are shown. Probability density functions are retrieved using only optical indices (red distributions) and both ultraviolet and optical indices (blue distributions). The blue shaded region represents the confidence interval corresponding to the 16-84 per cent percentile range of the PDF. The solid green line corresponds to the true value of $\mathrm{age}_{r}=4.31 \mathrm{Gyr}$ age $_{r}=4.44 \mathrm{Gyr}$, and age $r=3.94 \mathrm{Gyr}$, respectively; the dashed vertical red (blue) line indicates the median value of the corresponding distribution.

role of ultraviolet indices in helping to constrain light-weighted ages at different values of $S / N_{I, \mathrm{obs}}$.

For illustration, we show in Figs. 13 and 14 how the PDFs of age $_{r}$ and age $_{u}$ vary when using only optical or when using all available indices. We choose as examples three galaxies with different spectral types as parametrized by their value of $\mathrm{D}_{\mathrm{n}} 4000 \simeq[1.1,1.3,1.5]$ and two $S / N_{I, \mathrm{obs}}=[10,30]$. There is a clear improvement in constraining age $_{u}$ using ultraviolet indices, while their roles are almost negligible in constraining age $_{r}$.

Obviously the value of $S / N_{I, \mathrm{obs}}$ always plays a role, with an expected narrowing of the PDF width around the true value as $S / N_{I, \text { obs }}$ increases. However, and more interestingly, there is a noticeable difference as a function galaxy spectral type. For values of $\mathrm{D}_{\mathrm{n}} 4000 \gtrsim 1.5$, there is no improvement in constraining age $_{u}$ or age $_{r}$ by adding the information that comes from the bluer region of the spectrum. This result holds at both values of $S / N_{I, \text { obs }}$ considered, hinting at the physical interpretation that when a galaxy is truly old, the information provided by the ultraviolet and optical indices coincides. On the contrary, for values of $\mathrm{D}_{\mathrm{n}} 4000 \lesssim 1.5$ and even at $S / N_{I, \mathrm{obs}}=10$, the role of ultraviolet indices in increasing our ability to constrain age $_{u}$ is relatively clear. Their use helps in retrieving the true value and in reducing the uncertainty in the measurement. Even at $S / N_{I, \text { obs }}=30$, the use of optical indices alone cannot compete with the gain of information introduced by the bluer part of the spectrum (see middle panels in Fig. 14, as an example).

Moving to the general picture, in Figs. 15 and 16 we show the systematic relative deviations between true and measured values of age $\mathrm{e}_{r}$ and age $\mathrm{e}_{u}$ (using all available spectral indices) as a function of $D_{n} 4000$ values, respectively. Each point on these plots is color-coded according to the errors $\sigma_{\mathrm{obs}}$ as obtained from the PDF distribution. We find no significant systematic deviations

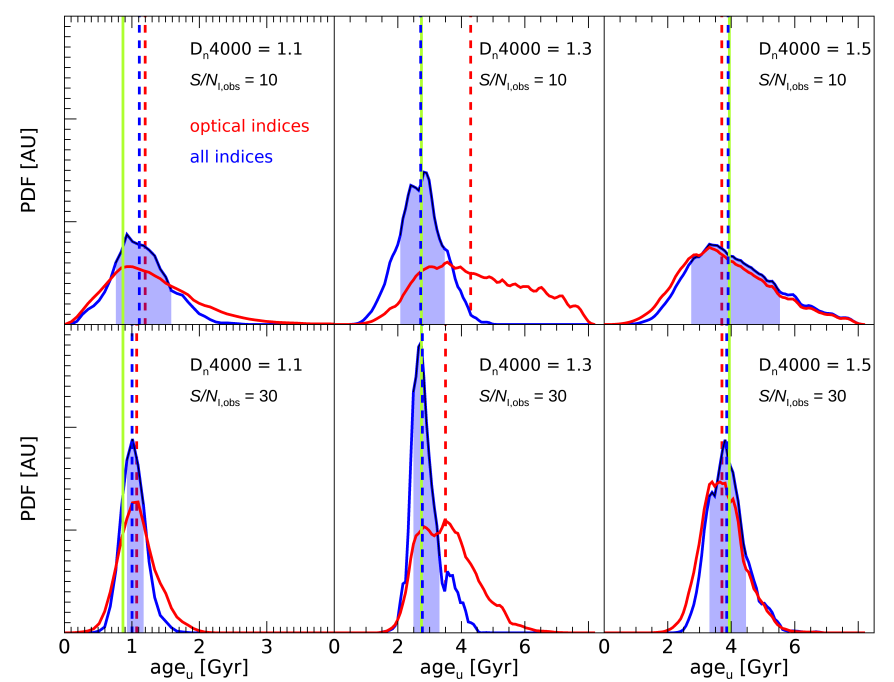

Fig. 14. As in Fig. 13, but for age $e_{u}$. The solid green line corresponds to the true value of age $e_{u}=0.87 \mathrm{Gyr}$, age $_{u}=2.74 \mathrm{Gyr}$, and age $e_{u}=$ $3.92 \mathrm{Gyr}$, respectively; the dashed vertical red (blue) line indicates to the median value of the corresponding distribution.

between true and measured values, as the median value of the distribution is consistent with zero.

We observe a clear trend in increasing $\sigma_{\text {obs }}$ as a function of increasing $\mathrm{D}_{\mathrm{n}} 4000$ values, both for $\mathrm{age}_{r}$ and $\mathrm{age}_{u}$ values. The trend is more obvious at $S / N_{I, \text { obs }}=10$, but persists as $S / N_{I, \text { obs }}$ increases, even as the actual typical values reached by $\sigma_{\text {obs }}$ decrease. This is not surprising as the spectral evolution proceeds more slowly as a stellar population ages (i.e., for larger values of $D_{n} 4000$ ), so that at older ages it takes larger absolute age differences to produce the same variation in observable quantities than at younger ages. In general, the relative (fractional) precision of age determination remains roughly constant with age. In fact, we find that $\sigma_{\text {obs }} /$ age $_{r, u}$ is $\sim 20 \%, \sim 15 \%$, and $\sim 10 \%$ for $S / N_{I, \text { obs }}=[10,20,30]$ over the full age range, respectively.

\subsubsection{Results: $\Delta$ (age)}

In this section we move to estimate the quantity $\Delta$ (age) for our observed galaxies using the method described in Sect. 5.2. The value of this physical parameter is directly computed for each model in the library and its full PDF is estimated from the Bayesian analysis ${ }^{1}$.

In Fig. 17 we plot the PDF of $\Delta$ (age), calculated by means of either only optical or all available indices at different $S / N_{I, \text { obs }}=[10,30]$ and various values of $\mathrm{D}_{\mathrm{n}} 4000 \simeq$ $[1.1,1.3,1.5]$. Similarly to age $_{u}$, the role of ultraviolet indices in improving the retrieval of the true value of $\Delta$ (age) is significant, both in the accuracy and the precision of the estimate. At higher $D_{n} 4000$ values, $\Delta$ (age) is intrinsically lower, and equally constrained using only optical or all indices available; however, the complexity of SFHs at lower $D_{n} 4000$ values results in differences between $u$ - and $r$-band light-weighted ages, which are actually more accurately defined using bluer indices. More importantly, the use of ultraviolet indices reduces the ambiguity of the peak at $\Delta$ (age) $\simeq 0$, which is present when only

1 That is, we do not estimate $\Delta$ (age) from the difference of the fiducial estimates of age and $_{r}$ age $e_{u}$, but we treat $\Delta$ (age) as a proper physical parameter in the models. 


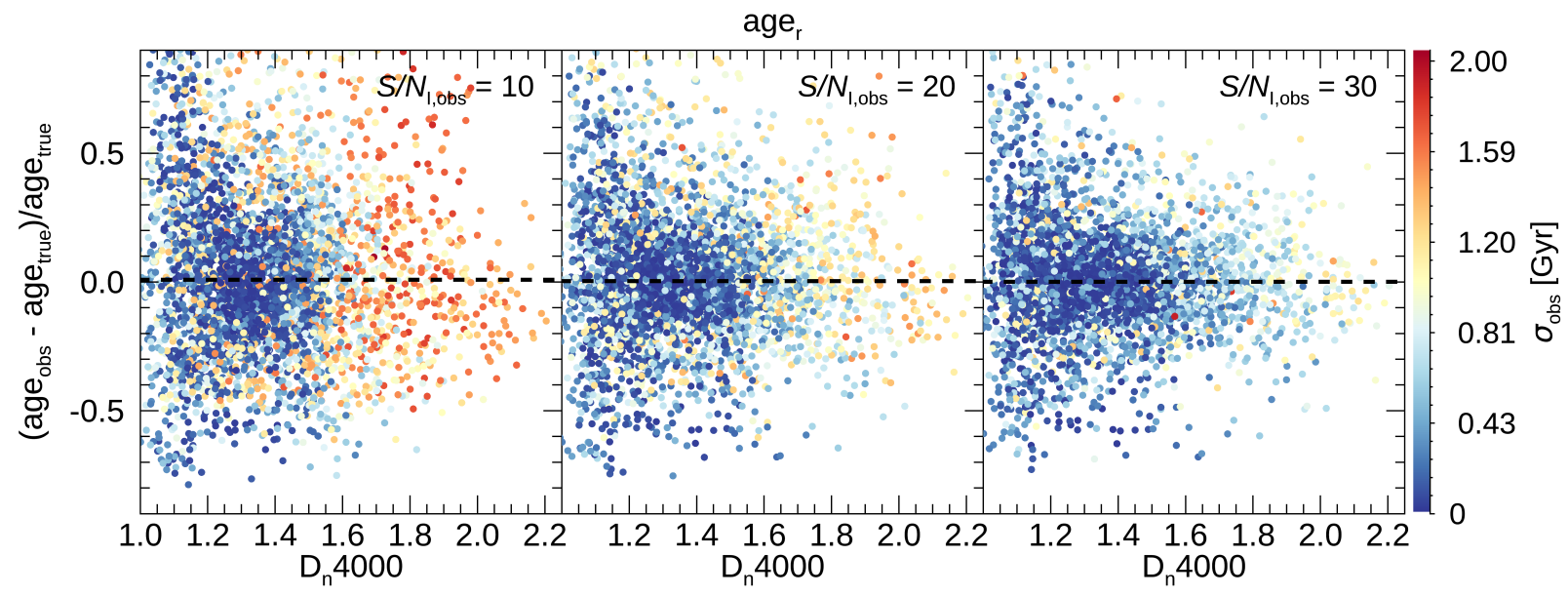

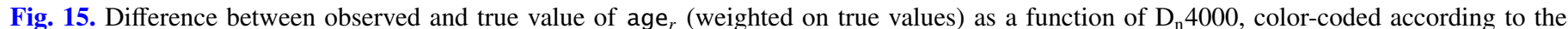
observed errors retrieved from PDFs. Galaxies are observed at $z=0.55$ and $S / N_{I, \mathrm{obs}}=[10,20,30]$ (from left to right). The black horizontal dashed line corresponds to the median value of the distribution.

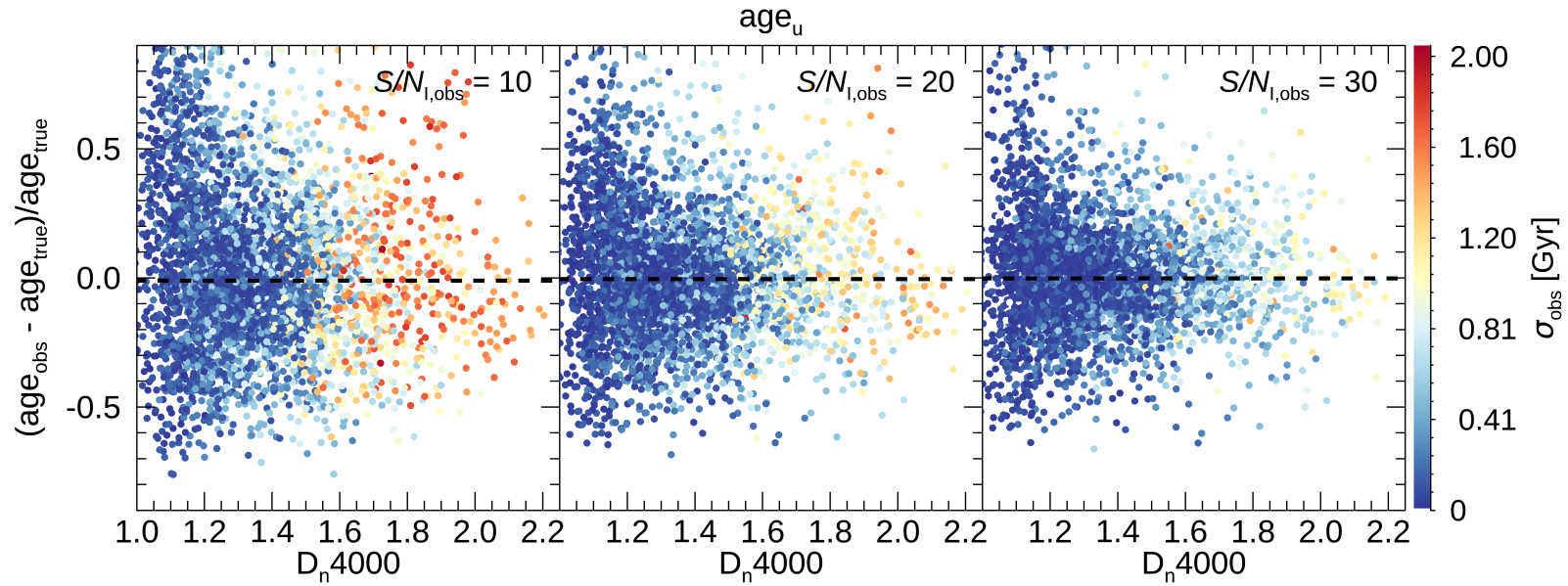

Fig. 16. As in Fig. 15, but for age .

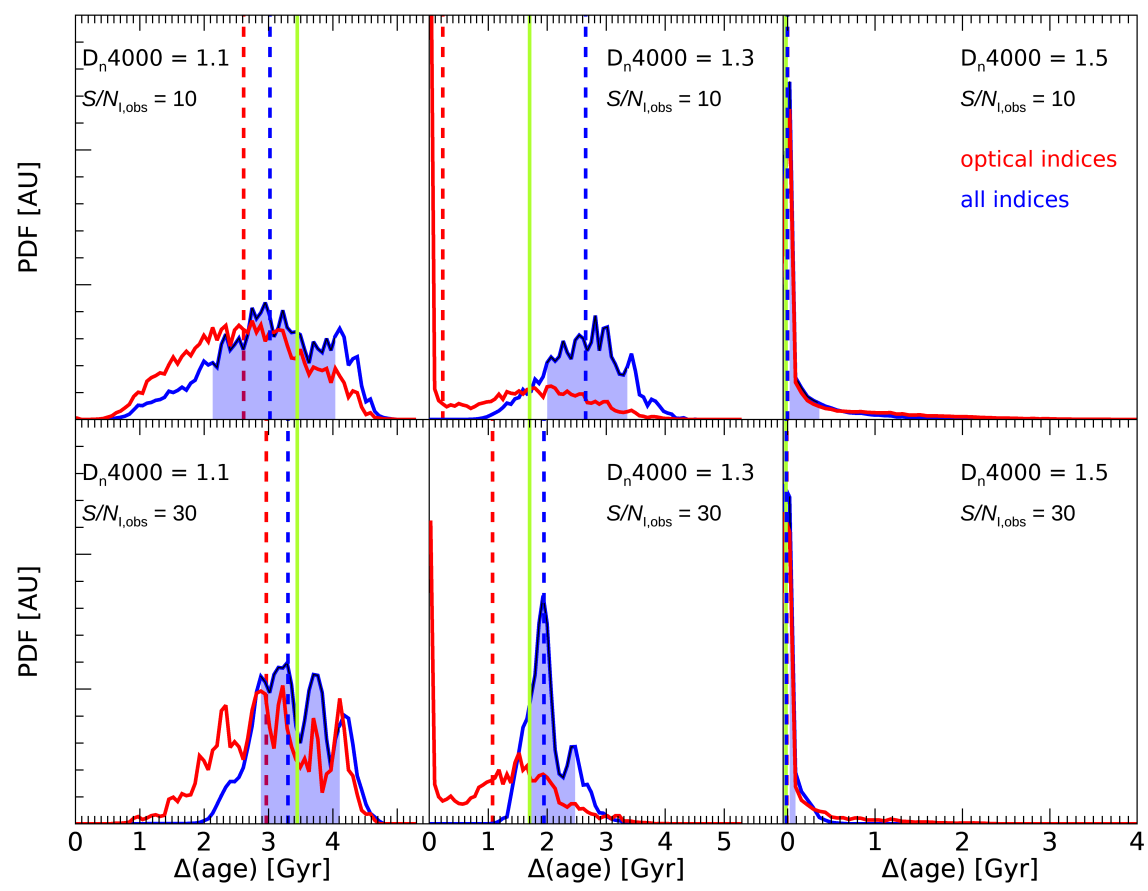

Fig. 17. As in Fig. 13, but for $\Delta$ (age). The solid green line corresponds to the true value of $\Delta$ (age) $=3.45 \mathrm{Gyr}, \Delta(\mathrm{age})=1.70 \mathrm{Gyr}$, and $\Delta($ age $)=0.02$ Gyr, respectively. 


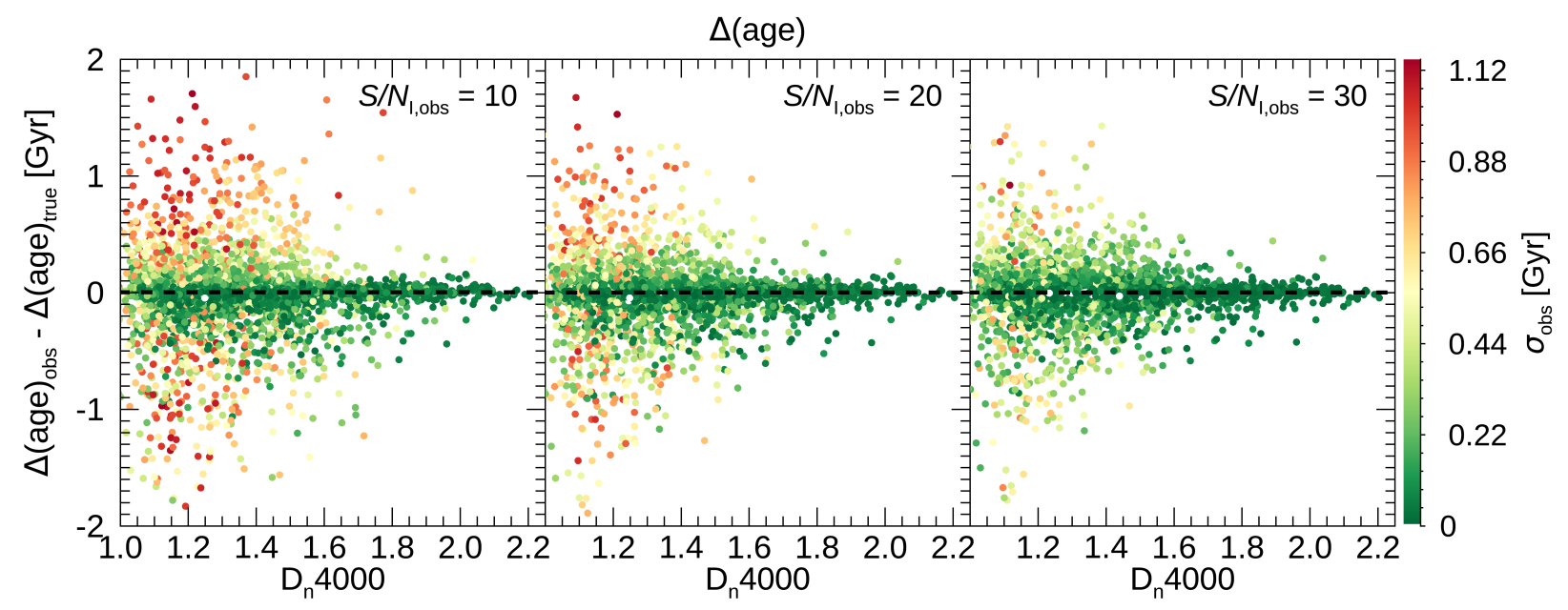

Fig. 18. Difference between observed and true value of $\Delta$ (age) as a function of $D_{n} 4000$, color-coded according to the observed errors retrieved from PDFs. Galaxies are observed at $z=0.55$ and $S / N_{I, \mathrm{obs}}=[10,20,30]$ (from left to right). The black horizontal dashed line corresponds to median value of the distribution.

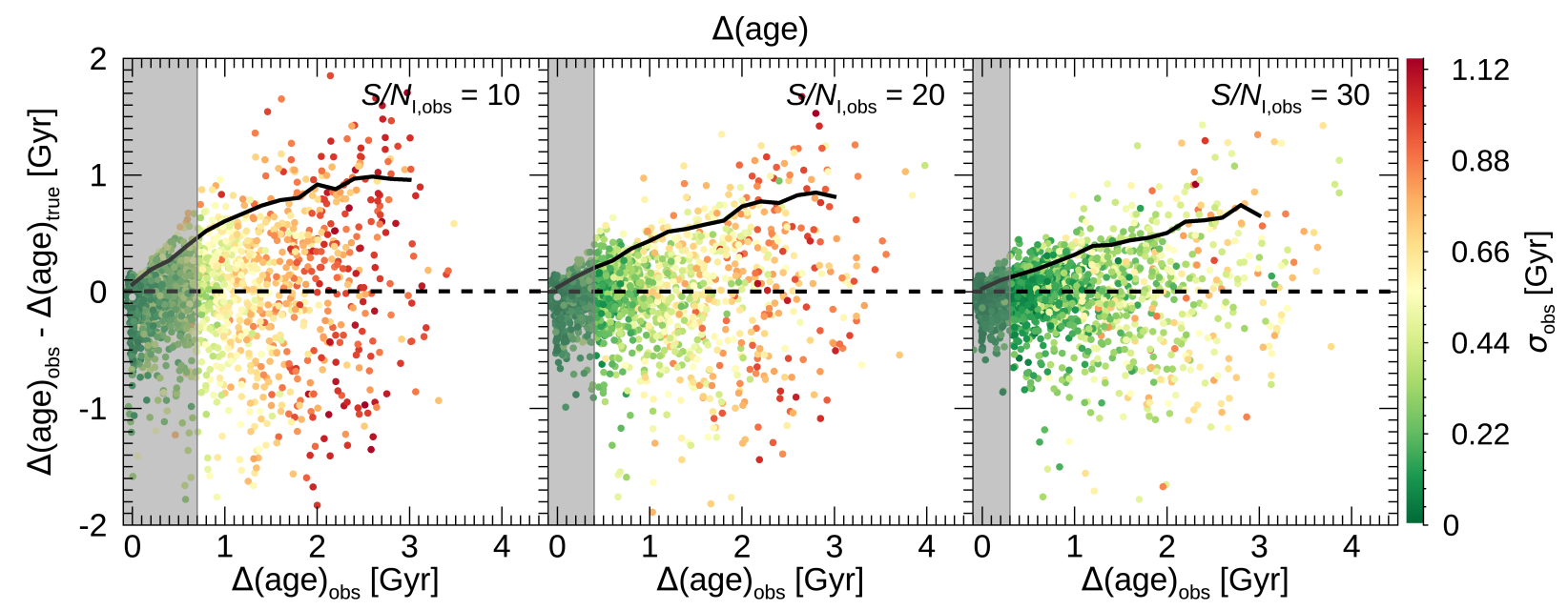

Fig. 19. Difference between observed and true values of $\Delta$ (age) as a function of $\Delta$ (age) ${ }_{\text {obs }}$, color-coded according to the observed errors retrieved from PDFs. Galaxies are observed at $z=0.55$ and $S / N_{I, \mathrm{obs}}=[10,20,30]$ (from left to right). The black horizontal dashed line corresponds to the median value of $\Delta$ (age $)_{\text {obs }}-\Delta$ (age $)_{\text {true }}$, while the black solid line represents the trend of observed errors at various values of $\Delta$ (age) ${ }_{\text {obs }}$. The gray shaded area represents the region compatible with $\Delta$ (age $)_{\mathrm{obs}}=0$.

optical indices are used (see e.g., middle panels in Fig. 17). Indeed, the information present in the bluer part of the spectrum allows us to prove the coexistence within the galaxy of a younger and older stellar population, information that would be irretrievable when looking only at redder wavelengths (Vazdekis et al. 2016).

In Fig. 18 we show our ability to recover the true value of $\Delta$ (age) as a function of $\mathrm{D}_{\mathrm{n}} 4000$ at $S / N_{I, \text { obs }}=[10,20,30]$. Each point on this plot is color-coded according to $\sigma_{\text {obs }}$ as obtained from the PDF. The systematic error in recovered $\Delta$ (age) values is negligible even at the lower $S / N_{I, \text { obs }}$. Furthermore, the scatter around the true value of $\Delta$ (age) is higher at lower $D_{n} 4000$ values and decreases at higher $S / N_{I, \text { obs }}$. A similar trend holds for the value of $\sigma_{\mathrm{obs}}$. It is worth noting that if $\mathrm{D}_{\mathrm{n}} 4000>1.5$, where the expected values of $\Delta$ (age) are intrinsically low, the ability of our methodology to retrieve the true value with small uncertainties is remarkable. On the other hand, the higher observational uncertainties $\sigma_{\text {obs }}$ at $\mathrm{D}_{\mathrm{n}} 4000<1.5$ are associated with the fact that this spectral feature is not an unambiguous indicator for $\Delta$ (age). In the region where $D_{n} 4000<1.5$, there are galaxies whose intrinsic $\Delta$ (age) values may be relatively large. The presence of higher scatter and higher observational errors is therefore the consequence of having galaxies whose PDFs are centered on intrinsically higher values of $\Delta$ (age) with broader PDFs. This is confirmed by plotting in Fig. 19 the absolute difference between observed and true values of $\Delta$ (age) as a function of the observed value of $\Delta$ (age), again color-coded as in Fig. 18. In this plot, higher values of $\Delta$ (age) have higher observational errors (i.e., broader PDFs). When $S / N_{I, \text { obs }}$ increases, the precision in the determination of $\Delta$ (age) increases (i.e., lower observational errors), as well as its accuracy (i.e., lower scatter around zero). However, even at lower $S / N_{I \text {,obs }}$ the information provided by the Bayesian analysis outperforms the coarse inference that classical diagnostics such as those discussed in Sect. 3 can provide.

Figure 19 clearly shows that, irrespective of $D_{n} 4000$ values, if the expected values of $\Delta$ (age) are intrinsically low, the ability to retrieve the true value with small uncertainties is remarkable $\left(\sigma_{\mathrm{obs}} \sim 0.1 \mathrm{Gyr}\right)$. This means that even at $S / N_{I, \mathrm{obs}}=10$ we are able not only to detect significant differences of $\Delta$ (age) values, 
but also to assert with reliable accuracy when $\Delta$ (age) is consistent with zero.

The importance of $\Delta$ (age) as a proxy to infer the presence of a significant spread in age between different stellar populations in individual galaxies relies on our ability to significantly determine a cutoff for defining a nonzero value of this parameter. Taking advantage of the Bayesian analysis and the full information provided by the PDF allows us to identify the values of $\Delta$ (age) that are robustly different from zero at different $S / N_{I, \text { obs }}$. Therefore, we divide the observed galaxies into bins of $0.1 \mathrm{Gyr}$ in true $\Delta$ (age) values and evaluate for each galaxy the area of the PDF that is below $\Delta$ (age) $<0.1 \mathrm{Gyr}$, a value that we define to be the threshold under which the measurement is compatible with $\Delta$ (age) $=0$ Gyr. In each bin of $\Delta$ (age), we then calculate the average value of the probability of being compatible with zero considering each single galaxy in that bin. We define as a reliable cutoff the bin at which the average probability is less than $10 \%$, finding $\Delta$ (age) $=0.7 \mathrm{Gyr}$ at $S / N_{I, \text { obs }}=$ $10, \Delta$ (age) $=0.4 \mathrm{Gyr}$ at $S / N_{I, \mathrm{obs}}=20$, and $\Delta$ (age) $=0.3 \mathrm{Gyr}$ at $S / N_{I, \text { obs }}=30$. This is the gray shaded area shown in Fig. 19.

Combining this information with that in Fig. 5, at the highest $\mathrm{S} / \mathrm{N}$ this analysis allows us to be sensitive to secondary episodes of star formation up to an age of $\sim 0.1 \mathrm{Gyr}$ for stellar populations older than $\sim 1.5 \mathrm{Gyr}$, pushing up to an age of $\sim 1 \mathrm{Gyr}$ for stellar populations older than $\sim 5 \mathrm{Gyr}$.

By means of Bayesian analysis and the availability of ultraviolet indices, we have been able to drastically reduce the uncertainties on the differences of $u$ - and $r$-band light-weighted ages, making this parameter suitable for inferring physical properties in individual galaxies. Moreover, the overall information contained in the PDF of $\Delta$ (age) allows us to properly distinguish between galaxies that display a coexistence of widely different stellar populations from those that present a more homogeneous, nearly coeval stellar population.

\section{Summary and conclusions}

The extragalactic community is on the eve of a new era in which large-field, high-multiplexing spectrographs with a remarkable wavelength span, mounted on four- to eight-meter-class telescopes, will allow the collection of moderately high-S/N spectra for tens of thousands of galaxies at intermediate to high redshift, encompassing both their rest-frame ultraviolet and optical range. While the ultimate science goal of our efforts in analyzing galaxy stellar continuum is to reconstruct the star formation and chemical enrichment history of a given galaxy, we must be aware that both theoretical limitations (intrinsic degeneracies) and technical limitations (chiefly the limited $\mathrm{S} / \mathrm{N}$ that can be attained in these surveys) will force us to focus on an essential but significant characterization.

In this work we concentrate in particular on the potential given by the simultaneous ultraviolet and visible rest-frame coverage, despite a moderate $\mathrm{S} / \mathrm{N}(\sim 10-30)$. Starting from the consideration that stellar populations of different ages affect the spectrum at different wavelengths differently, we investigate how the difference $\Delta$ (age) between the light-weighted age in the rest-frame $u$ - and $r$-band can be used as a simple tool to discern the complexity of SFHs in galaxies. Furthermore, we find that $\Delta$ (age) is essentially a diagnostic of coexistence of old (ultraviolet-faint) stellar populations and young (ultraviolet-

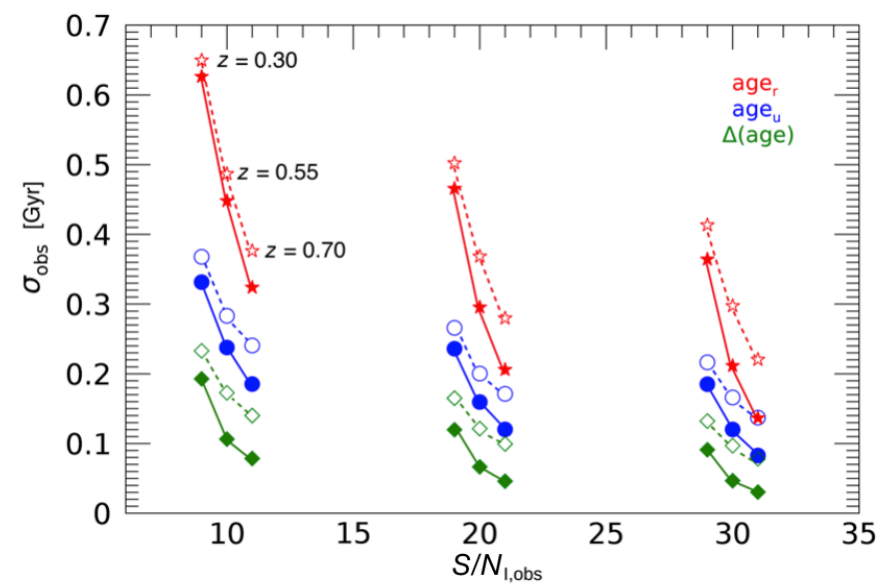

Fig. 20. Median value of observational errors on age $r$ (red stars), age a $_{u}$ (blue circles), and $\Delta$ (age) (green diamonds) as a function of $S / N_{I, \text { obs }}$. Filled symbols correspond to Bayesian analysis where all measured indices were used, while empty symbols correspond to PDFs retrieved using only optical indices.

bright) stellar populations. Using a vast spectral library of SFHs, including both smooth and bursty ones, we demonstrate that a large $\Delta$ (age) is a symptom of either a smooth but very extended SFH or of a SFH with widely spaced peaks (e.g., an old "secular" peak and a recent burst). Although $\Delta$ (age) cannot discriminate between these two scenarios by itself, it has the great advantage of being robust against degeneracies and largely independent of the actual implementation of SFHs in models, provided that enough complexity is included.

Based on realistic simulations of WEAVE-StePS spectra, we assessed our future ability to measure $\Delta$ (age) at different redshifts and $\mathrm{S} / \mathrm{Ns}$, and its consequent diagnostic power. More specifically, we simulated spectra at three different redshifts $z=[0.3,0.55,0.7]$ and three different $\mathrm{S} / \mathrm{N}$ in the observed-frame $I$-band, $S / N_{I, \text { obs }}=[10,20,30]$. We took into account the typical transmission, efficiency, and noise derived from the characteristic of the observing site, telescope, fibers, spectrograph, and CCDs for the low-resolution configuration adopted by StePS for the WEAVE spectrograph on the WHT in La Palma.

We adopted the Bayesian approach developed by Gallazzi et al. (2005) and confronted the spectral absorption indices as measured in the simulated spectra with those in our vast library of models to obtain the marginalized PDF for $\Delta$ (age), as well

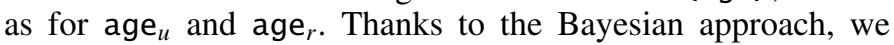
obtained remarkable accuracy and precision in our estimates, in particular when indices in both the ultraviolet and visible range are combined. In Fig. 20 we show the trend of the median uncertainty in age $r$, age $_{u}$, and $\Delta$ (age) at different $S / N_{I, \text { obs }}$ as a function of redshift $z=[0.3,0.55,0.7]$. Errors on every parameter decrease as both $S / N_{I, \text { obs }}$ and redshift increase. While the former behavior is expected, the improvement at higher redshift can be ascribed to the increasing number of ultraviolet spectral features that become available, and the better $\mathrm{S} / \mathrm{N}$ at which they are measured due to the shift of the spectrum toward the I-band where $\mathrm{S} / \mathrm{N}$ is fixed (see Sect. 4.2). Under the assumption of the existence of an old underlying stellar population (as is the case for the massive galaxies we are mainly going to target in StePS), these errors translate into the ability to identify secondary episodes of star formation younger than $\sim 1 \mathrm{Gyr}$ for stellar populations older than $\sim 5 \mathrm{Gyr}$, or episodes younger 
than $\sim 0.1 \mathrm{Gyr}$ for stellar populations older than $\sim 1.5 \mathrm{Gyr}$. In this sense, $\Delta$ (age) and age ${ }_{r}$ together present a powerful diagnostic of rejuvenation episodes in old galaxies, and, conversely, allow us to check for the truly passive evolution of old stellar populations with a much greater sensitivity than age $_{r}$ alone.

Finally, it is worth stressing that the analysis presented in this paper can easily be adapted for similar instruments and surveys and can be tested with different stellar population libraries, thus allowing for the benchmarking of both instrumental effects and theoretical models and assumptions.

Acknowledgements. We thank the anonymous referee for carefully reading the manuscript and for the suggestions that helped us to improve the way we presented our results. We wish to thank M. Irwin for very useful discussions and valuable suggestions. L. C. wish to acknowledge financial support from Premiale 2015 MITiC, program 1.05.06.10 and Comunidad de Madrid under Atracción de Talento grant 2018-T2/TIC-11612. A. I., S. Z., M. L., A. G., A. M. and C. T. acknowledge the financial support from the INAF PRIN-SKA 2017 program 1.05.01.88.04.ESKAPE-HI. I. L. acknowledges financial support from INAF-WEAVE funds, program 1.05.03.04.05. R. G. B. acknowledges support from the Spanish Ministry of Economy and Competitiveness through grant 205 AYA2016-77846-P and the State Agency for Research of the Spanish MCIU through the "Center of Excellence Severo Ochoa" award to 2017 the Instituto de Astrofísica de Andalucía (SEV-2017-0709). A. F. M. has received financial support through the Postdoctoral Junior Leader Fellowship Programme from "La Caixa" Banking Foundation (LCF/BQ/LI18/11630007). B. V. acknowledges the financial support from INAF Main Stream 2018 (P. I.: B. Vulcani).

\section{References}

Abramson, L. E., Gladders, M. D., Dressler, A., et al. 2015, ApJ, 801, L12 Baldry, I. K., Glazebrook, K., Brinkmann, J., et al. 2004, ApJ, 600, 681 Baldry, I. K., Balogh, M. L., Bower, R. G., et al. 2006, MNRAS, 373, 469 Balogh, M. L., Morris, S. L., Yee, H. K. C., Carlberg, R. G., \& Ellingson, E. 1999, ApJ, 527, 54

Bamford, S. P., Nichol, R. C., Baldry, I. K., et al. 2009, MNRAS, 393, 1324

Behroozi, P. S., Wechsler, R. H., \& Conroy, C. 2013, ApJ, 770, 57

Benn, C. R., \& Ellison, S. L. 1998, New Astron. Rev., 42, 503

Blanton, M. R., \& Moustakas, J. 2009, ARA\&A, 47, 159

Blanton, M. R., Hogg, D. W., Bahcall, N. A., et al. 2003, ApJ, 594, 186

Bruzual, G., \& Charlot, S. 2003, MNRAS, 344, 1000

Cappellari, M. 2017, MNRAS, 466, 798

Cappellari, M., \& Emsellem, E. 2004, PASP, 116, 138

Carnall, A. C., McLure, R. J., Dunlop, J. S., \& Davé, R. 2018, MNRAS, 480, 4379

Chabrier, G. 2003, ApJ, 586, L133

Charlot, S., \& Fall, S. M. 2000, ApJ, 539, 718

Chauke, P., van der Wel, A., Pacifici, C., et al. 2019, ApJ, 877, 48

Cid Fernandes, R., Mateus, A., Sodré, L., Stasińska, G., \& Gomes, J. M. 2005, MNRAS, 358, 363

Costantin, L., Méndez-Abreu, J., Corsini, E. M., et al. 2017, A\&A, 601, A84

Costantin, L., Méndez-Abreu, J., Corsini, E. M., et al. 2018, A\&A, 609, A132

Daddi, E., Renzini, A., Pirzkal, N., et al. 2005, ApJ, 626, 680

Dalton, G., Trager, S. C., Abrams, D. C., et al. 2012, in Ground-based and Airborne Instrumentation for Astronomy IV, SPIE Conf. Ser., 8446, 84460P

Dalton, G., Ham, S. J., Trager, S., et al. 2016, SPIE Conf. Ser., 9913, 99132X

Davidzon, I., Ilbert, O., Laigle, C., et al. 2017, A\&A, 605, A70

de Jong, R. S., Agertz, O., Berbel, A. A., et al. 2019, The Messenger, 175, 3

de Lorenzo-Cáceres, A., Falcón-Barroso, J., \& Vazdekis, A. 2013, MNRAS, 431, 2397

Diemer, B., Sparre, M., Abramson, L. E., \& Torrey, P. 2017, ApJ, 839, 26

Falcón-Barroso, J., Sánchez-Blázquez, P., Vazdekis, A., et al. 2011, A\&A, 532, A95

Fanelli, M. N., O’Connell, R. W., Burstein, D., \& Wu, C.-C. 1992, ApJS, 82, 197

Ferré-Mateu, A., Sánchez-Blázquez, P., Vazdekis, A., \& de la Rosa, I. G. 2014, ApJ, 797, 136

Gallazzi, A., Charlot, S., Brinchmann, J., White, S. D. M., \& Tremonti, C. A. 2005, MNRAS, 362, 41

Gallazzi, A., Bell, E. F., Zibetti, S., Brinchmann, J., \& Kelson, D. D. 2014, ApJ, 788, 72

Gargiulo, A., Bolzonella, M., Scodeggio, M., et al. 2017, A\&A, 606, A113
Girardi, L., Bressan, A., Bertelli, G., \& Chiosi, C. 2000, A\&AS, 141, 371 Gladders, M. D., Oemler, A., Dressler, A., et al. 2013, ApJ, 770, 64 Guglielmo, V., Poggianti, B. M., Vulcani, B., et al. 2019, A\&A, 625, A112 Hahn, C., Blanton, M. R., Moustakas, J., et al. 2015, ApJ, 806, 162

Haines, C. P., Iovino, A., Krywult, J., et al. 2017, A\&A, 605, A4 Iovino, A., Cucciati, O., Scodeggio, M., et al. 2010, A\&A, 509, A40 Jørgensen, I., \& Chiboucas, K. 2013, AJ, 145, 77

Kauffmann, G., Heckman, T. M., White, S. D. M., et al. 2003, MNRAS, 341, 33

Kauffmann, G., White, S. D. M., Heckman, T. M., et al. 2004, MNRAS, 353, 713

Kaviraj, S., Peirani, S., Khochfar, S., Silk, J., \& Kay, S. 2009, MNRAS, 394, 1713

Knobel, C., Lilly, S. J., Iovino, A., et al. 2009, ApJ, 697, 1842

Kovač, K., Lilly, S. J., Knobel, C., et al. 2010, ApJ, 718, 86

Krywult, J., Tasca, L. A. M., Pollo, A., et al. 2017, A\&A, 598, A120

La Barbera, F., Pasquali, A., Ferreras, I., et al. 2014, MNRAS, 445, 1977

Leja, J., Johnson, B. D., Conroy, C., van Dokkum, P. G., \& Byler, N. 2017, ApJ, 837,170

Lonoce, I., Longhetti, M., Saracco, P., Gargiulo, A., \& Tamburri, S. 2014, MNRAS, 444, 2048

López Fernández, R., González Delgado, R. M., Pérez, E., et al. 2018, A\&A, 615, A27

Maraston, C., Nieves Colmenárez, L., Bender, R., \& Thomas, D. 2009, A\&A, 493, 425

Marigo, P., Bressan, A., Nanni, A., Girardi, L., \& Pumo, M. L. 2013, MNRAS, 434, 488

Martins, L. P., González Delgado, R. M., Leitherer, C., Cerviño, M., \& Hauschildt, P. 2005, MNRAS, 358, 49

Méndez-Abreu, J., Sánchez-Janssen, R., Aguerri, J. A. L., Corsini, E. M., \& Zarattini, S. 2012, ApJ, 761, L6

Méndez-Abreu, J., Costantin, L., Aguerri, J. A. L., de Lorenzo-Cáceres, A., \& Corsini, E. M. 2018, MNRAS, 479, 4172

Morelli, L., Corsini, E. M., Pizzella, A., et al. 2015, MNRAS, 452, 1128

Nelson, D., Pillepich, A., Genel, S., et al. 2015, Astron. Comput., 13, 12

Ocvirk, P., Pichon, C., Lançon, A., \& Thiébaut, E. 2006, MNRAS, 365, 74

Onodera, M., Renzini, A., Carollo, M., et al. 2012, ApJ, 755, 26

Pacifici, C., Kassin, S. A., Weiner, B. J., et al. 2016, ApJ, 832, 79

Pandya, V., Brennan, R., Somerville, R. S., et al. 2017, MNRAS, 472, 2054

Pérez-González, P. G., Rieke, G. H., Villar, V., et al. 2008, ApJ, 675, 234

Poggianti, B. M., Fasano, G., Bettoni, D., et al. 2009, ApJ, 697, L137

Sánchez-Blázquez, P., Peletier, R. F., Jiménez-Vicente, J., et al. 2006, MNRAS, 371,703

Sandage, A. 1986, A\&A, 161, 89

Sarzi, M., Falcón-Barroso, J., Davies, R. L., et al. 2006, MNRAS, 366, 1151

Schaye, J., Crain, R. A., Bower, R. G., et al. 2015, MNRAS, 446, 521

Schiavon, R. P., Faber, S. M., Konidaris, N., et al. 2006, ApJ, 651, L93

Serven, J., Worthey, G., \& Briley, M. M. 2005, ApJ, 627, 754

Spilker, J., Bezanson, R., Barišić, I., et al. 2018, ApJ, 860, 103

Takada, M., Ellis, R. S., Chiba, M., et al. 2014, PASJ, 66, R1

Thomas, D., Maraston, C., Bender, R., \& Mendes de Oliveira, C. 2005, ApJ, 621, 673

Thomas, D., Maraston, C., Schawinski, K., Sarzi, M., \& Silk, J. 2010, MNRAS, 404, 1775

Tojeiro, R., Wilkins, S., Heavens, A. F., Panter, B., \& Jimenez, R. 2009, ApJS, 185,1

Trager, S. C., Faber, S. M., Worthey, G., \& González, J. J. 2000, AJ, 120, 165

van der Wel, A., Noeske, K., Bezanson, R., et al. 2016, ApJS, 223, 29

Vazdekis, A., Koleva, M., Ricciardelli, E., Röck, B., \& Falcón-Barroso, J. 2016, MNRAS, 463, 3409

Worthey, G., Faber, S. M., Gonzalez, J. J., \& Burstein, D. 1994, ApJS, 94, 687

Wright, E. L. 2006, PASP, 118, 1711

Wu, P.-F., van der Wel, A., Gallazzi, A., et al. 2018, ApJ, 855, 85

York, D. G., Adelman, J., Anderson, Jr., J. E., et al. 2000, AJ, 120, 1579

Zahid, H. J., Dima, G. I., Kudritzki, R.-P., et al. 2014, ApJ, 791, 130

Zibetti, S., Gallazzi, A. R., Ascasibar, Y., et al. 2017, MNRAS, 468, 1902

1 INAF - Osservatorio Astronomico di Brera, Via Brera 28, 20121 Milano, Italy e-mail: luca. costantin@inaf .it

2 Centro de Astrobiología (CSIC-INTA), Ctra de Ajalvir km 4, Torrejón de Ardoz 28850 Madrid, Spain

3 INAF - Osservatorio Astrofisico di Arcetri, Largo Enrico Fermi 5, 50125 Firenze, Italy

4 INAF - Osservatorio Astronomico di Capodimonte, Salita Moiariello 16, 80131 Napoli, Italy 
5 Department of Astronomy and Astrophysics, University of Chicago, Chicago, IL 60637, USA

${ }^{6}$ Isaac Newton Group of Telescopes, Apartado 321, 38700 Santa Cruz de La Palma, Canary Islands, Spain

7 Instituto de Astrofísica de Canarias, Calle Vía Láctea s/n, 38200 La Laguna, Tenerife, Spain

8 Departamento de Astrofísica, Universidad de La Laguna, Calle Astrofísico Francisco Sánchez s/n, 38205 La Laguna, Tenerife, Spain

${ }^{9}$ INAF - Osservatorio di Astrofisica e Scienza dello Spazio di Bologna, Via Gobetti 93/3, 40129 Bologna, Italy

${ }_{10}$ Department of Physics, University of Oxford, Keble Road, OX1 3RH Oxford, UK

11 STFC-RALSpace, Rutherford Appleton Laboratory, Didcot OX11 OQX, UK

12 Institut de Ciencies del Cosmos (ICCUB), Universitat de Barcelona (IEEC-UB), 02028 Barcelona, Spain

13 Centre for Astrophysics \& Supercomputing, Swinburne University of Technology, Hawthorn, VIC 3122, Australia
14 Instituto de Astrofísica de Andalucía (CSIC), PO Box 3004, 18080 Granada, Spain

15 INAF - IASF Milano, Via Bassini 15, 20133 Milano, Italy

16 Instituto de Astronomía y Ciencias Planetarias de Atacama, Universidad de Atacama, Copayapu 485, Copiapó, Chile

17 Kapteyn Astronomical Institute, University of Groningen, Postbus 800, 9700 Groningen, The Netherlands

18 School of Physics and Astronomy, University of Birmingham, Edgbaston, Birmingham B15 2TT, UK

19 Institute of Astronomy, University of Cambridge, Madingley Road, Cambridge CB3 0HA, UK

20 Dipartimento di Fisica e Astronomia “G. Galilei”, Università di Padova, Vicolo dell'Osservatorio 3, 35122 Padova, Italy

21 INAF - Osservatorio Astronomico di Padova, vicolo dell' Osservatorio 5, 35122 Padova, Italy

22 Departamento de Física Teórica, Universidad Autónoma de Madrid, 28049 Cantoblanco, Spain

23 University of Bologna - Department of Physics and Astronomy, Via Gobetti 93/2, 40129 Bologna, Italy 